\title{
TW Hya: SPECTRAL VARIABILITY, X-RAYS, AND ACCRETION DIAGNOSTICS
}

\author{
A. K. Dupree ${ }^{1}$, N. S. Brickhouse ${ }^{1}$, S. R. Cranmer ${ }^{1}$, G. J. M. Luna ${ }^{1,8}$, E. E. Schneider ${ }^{1,9}$, M. S. Bessell ${ }^{2}$, A. Bonanos ${ }^{3}$, \\ L. A. Crause ${ }^{4}$, W. A. Lawson ${ }^{5}$, S. V. Mallik ${ }^{6}$ and S. C. Schuler ${ }^{7}$ \\ ${ }^{1}$ Harvard-Smithsonian Center for Astrophysics, Cambridge, MA 02138, USA \\ ${ }^{2}$ Australian National Observatory, Mount Stromlo Observatory, Canberra, ACT 2611, Australia \\ ${ }^{3}$ Institute of Astronomy and Astrophysics, National Observatory of Athens, 15236 Athens, Greece \\ ${ }^{4}$ South African Astronomical Observatory, P.O. Box 9, Observatory 7935, Cape Town, South Africa \\ ${ }^{5}$ School of Physical, Environmental, and Math Sciences, University of New South Wales, Canberra, ACT 2600, Australia \\ ${ }^{6}$ Indian Institute of Astrophysics, Bangalore 560034, India \\ ${ }^{7}$ National Optical Astronomy Observatory, Tucson, AZ 85719, USA \\ Received 2011 November 21; accepted 2012 February 24; published 2012 April 17
}

\begin{abstract}
The nearest accreting T Tauri star, TW Hya was intensively and continuously observed over $\sim 17$ days with spectroscopic and photometric measurements from four continents simultaneous with a long segmented exposure using the Chandra satellite. Contemporaneous optical photometry from WASP-S indicates a 4.74 day period was present during this time. The absence of a similar periodicity in the $\mathrm{H} \alpha$ flux and the total X-ray flux which are dominated by accretion processes and the stellar corona, respectively, points to a different source of photometric variations. The $\mathrm{H} \alpha$ emission line appears intrinsically broad and symmetric, and both the profile and its variability suggest an origin in the post-shock cooling region. An accretion event, signaled by soft X-rays, is traced spectroscopically for the first time through the optical emission line profiles. After the accretion event, downflowing turbulent material observed in the $\mathrm{H} \alpha$ and $\mathrm{H} \beta$ lines is followed by $\mathrm{He}$ ( $(\lambda 5876$ ) broadening near the photosphere. Optical veiling resulting from the heated photosphere increases with a delay of $\sim 2 \mathrm{hr}$ after the X-ray accretion event. The response of the stellar coronal emission to an increase in the veiling follows $\sim 2.4 \mathrm{hr}$ later, giving direct evidence that the stellar corona is heated in part by accretion. Subsequently, the stellar wind becomes re-established. We suggest a model that incorporates the dynamics of this sequential series of events: an accretion shock, a cooling downflow in a supersonically turbulent region, followed by photospheric and later, coronal heating. This model naturally explains the presence of broad optical and ultraviolet lines, and affects the mass accretion rates determined from emission line profiles.
\end{abstract}

Key words: accretion, accretion disks - stars: individual (TW Hydrae) - stars: pre-main sequence - stars: variables: T Tauri, Herbig Ae/Be - stars: winds, outflows

Online-only material: color figures

\section{INTRODUCTION}

The young dwarf star TW Hya ${ }^{10}$ (CD -34 7151, TWA 1, HIP 53911) is arguably the closest accreting $\mathrm{T}$ Tauri star (Wichmann et al. 1998), and as such offers an opportunity to study the accretion process and properties of the stellar atmosphere and wind. Of particular interest is the fact that the accretion disk surrounding TW Hya is oriented almost "faceon" and the rotation axis of the star has a low inclination (Krist et al. 2000; Qi et al. 2004). Such an orientation places the polar regions of the star in full view where accretion of material from a surrounding circumstellar disk is thought to occur. Moreover, it is likely that open magnetic field structures exist at the poles, where a stellar wind may arise. TW Hya presents a bright target with rich scientific potential to unravel both the accretion process and its relation to coronal heating and accretion-driven winds from young stars.

TW Hya has been well studied and monitored with optical and ultraviolet spectra (Muzerolle et al. 2000; Alencar \& Batalha 2002; Ardila et al. 2002; Batalha et al. 2002; Dupree et al. 2005; Curran et al. 2011), exhibiting variations in emission line profiles

\footnotetext{
8 Current address: Instituto de Astronomia y Fisica del Espacio, (IAFE), Buenos Aires, Argentina.

9 Current address: Astronomy Department, University of Arizona, Tucson, AZ, USA.

${ }^{10} V=11.1 ;$ R.A. $=11^{\mathrm{h}} 01^{\mathrm{m}} 52^{\mathrm{s}} ;$ decl. $=-34^{\circ} 42^{\prime} 17^{\prime \prime} .03($ FK5 2000).
}

that are attributed principally to accretion and the presence of a stellar wind.

An X-ray spectrum of TW Hya obtained with the Chandra Observatory in 2000 first indicated very high densities that Kastner et al. (2002) attributed to the shock arising from material accreting from the surrounding circumstellar disk in magnetically channeled flows. A long (500 ks) pointing by the Chandra satellite with the HETG grating confirmed those densities, and differentiated the emission lines from the accretion shock and the stellar corona (Brickhouse et al. 2010). Importantly, the X-ray diagnostics revealed a large coronal volume resulting from heating by the shocked plasma. The Chandra spectrum, spectral diagnostics, and model from these definitive observations have been discussed elsewhere (Brickhouse et al. 2010). Here, we report on simultaneous and contemporaneous optical and infrared photometry and spectroscopy. In particular, the behavior of X-rays and the strength and profiles of the hydrogen and helium emission lines are investigated because simultaneous measures can elucidate their relationships.

These photometric and spectroscopic observations were obtained at a variety of sites that span times of the Chandra pointings. TW Hya is located at a declination of $-35^{\circ}$, making it accessible from the northern hemisphere several hours per night during transit, in addition to easy access from the southern hemisphere. Characteristics of the individual observations follow 
Table 1

Chandra ACIS-S/HETG Observations

\begin{tabular}{lcc}
\hline \hline ObsID & $\begin{array}{c}\text { JD Start } \\
(-2454100)\end{array}$ & $\begin{array}{c}\text { Exposure } \\
(\mathrm{ks})\end{array}$ \\
\hline 7435 & 46.986 & 152 \\
7437 & 57.519 & 160 \\
7436 & 60.146 & 162 \\
7438 & 63.156 & 23 \\
\hline
\end{tabular}

below. Table 1 contains a summary of the Chandra observations; Table 2 summarizes the optical campaign. Figure 1 displays the observations at various observatories in conjunction with the four segments of the Chandra pointings.

Photometry is addressed first (Section 2), in order to establish a photometric period for the star contemporaneously with the Chandra observations and the spectroscopy. In Section 3, the $\mathrm{X}$-ray measurements, deconstructed into a coronal and accretion component, are compared with the optical photometry to isolate accretion and flaring events. Section 4 summarizes the optical and infrared spectroscopy efforts. Subsequent sections address the variation of the $\mathrm{H} \alpha$ and $\mathrm{H} \beta$ fluxes from the monitoring program (Section 5) and an intensive high-resolution optical study of the $\mathrm{H} \alpha, \mathrm{H} \beta$, and $\mathrm{He}$ I profiles over three successive nights. The following sections detail the optical spectroscopic response to the X-ray accretion events and a stellar X-ray flare (Sections 6 and 7). The wind, as detected in the near-infrared line of He I is compared to previous measures and to the nearsimultaneous optical spectrum in Section 8. Section 9 contains the veiling parameters and explores their relation to the X-ray flux. A model incorporating our results is put forth in Section 10, and comparison with previous results is made in Section 11. Conclusions can be found in Section 12.

\section{PHOTOMETRIC OBSERVATIONS: SUPER WASP-S, SAAO, AND ASAS}

Photometry from the Super WASP-South program (Butters et al. 2010) spanned the Chandra observations, and a period can be sought from those data. The measurements, shown in the top panel of Figure 2, contained a flaring episode on JD 2454155 (Day 55). ${ }^{11}$ A period search on the non-flaring data (see Figure 2, lower panel) was made using the algorithm of Horne \& Baliunas (1986) and Scargle (1982), which accommodates unequally spaced data. A clear peak occurs in the periodogram corresponding to 4.744 days during this span of 19.2 days from JD 2454148.33 to JD 2454167.48. The False Event probability assigned to this period is less than $10^{-5}$. A long history of searches for a photometric period of TW Hya can be found in the literature (cf. Rucinski et al. 2008), which reveals values ranging from 1.3 to $\sim 6$ days. Periods around 4.74 days have been seen in $B$-band veiling (Batalha et al. 2002) and in some line intensity data (Alencar \& Batalha 2002) and been suggested by $B$-band photometry (Lawson \& Crause 2005). While the photometric variations might arise from the presence of accretion hot spots or rings on the stellar surface, or, alternately as suggested by Siwak et al. (2011), from optically thick plasma condensations in the inner accretion disk, a clear periodicity is confounded by flaring episodes. No long-term periodicity could be identified in Hipparcos photometry (Kastner et al. 1999), although Koen \& Eyer (2002) identified a 2.88 day period, which

\footnotetext{
11 Here the nomenclature "Day 55" represents the Julian Date minus an offset: JD-2454100
}

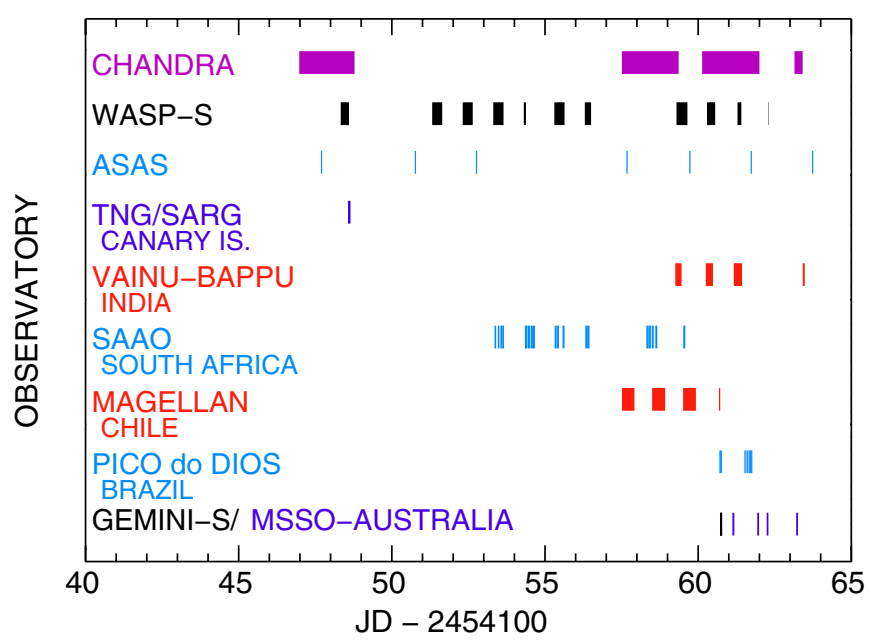

Figure 1. Overview of the dates of observations at the participating observatories and the Chandra pointings on TW Hya.

(A color version of this figure is available in the online journal.)

corresponds to the $V$-band photometry of Lawson \& Crause (2005). Continuous observations over a short time span appear to yield a reliable value of the period, but these values can differ one from another, and it is not clear what phenomenon or combination of effects is represented by a "period." The study by Rucinski et al. (2008) analyzing photometry from the $M i$ crovariability and Oscillations of STars (MOST) satellite during a continuous 11 day observation in 2007 yielded a periodicity of 3.7 days and the amplitude spectrum contained significant real components with periods longer than 0.5 days. The MOST observation followed the $\sim 19$ day WASP-S segments considered in this paper by $\sim 7$ days; however, the periodogram from the WASP-S data shows no peak at the period found from MOST. Rucinski et al. (2008) noted that in 2008, the oscillations appeared to move toward shorter periods, from 5-6 days to 3 days over a time interval of 10 days. A systematic period-shortening on timescales of weeks is confirmed in the 2009 MOST photometry as well (Siwak et al. 2011). Thus, the value found here is not inconsistent with the MOST measurements.

Precision velocities from spectroscopic measurements made in 2008 February-March suggested a period of 3.57 days (Huélamo et al. 2008), which was attributed to a cool spot at high latitudes. Rucinski et al. (2008) did not detect this period from MOST photometry made at the same time. The WASP photometry (Figure 2) also does not indicate a period of 3.57 days $\left(0.280\right.$ day $\left.^{-1}\right)$. It seems possible that the photometry traces variability related to the accretion process in some way. And so, we assume the period of 4.74 days as the fiducial period for comparison here because it was derived from WASP-S measurements spanning the time of the time of these X-ray and optical measurements. When the photometry of TW Hya is phased to the 4.74 day period (see Figure 3 ), variability on shorter timescales is also present. Such variability is typical of $\mathrm{T}$ Tauri stars.

Differential $V$-band photometry at several epochs was obtained at the Sutherland field station of the South African Astronomical Observatory (SAAO) with a $1 \mathrm{~m}$ telescope and a $1 \mathrm{k} \times 1 \mathrm{k}$ SITe charge-coupled device (CCD) at SAAO between 2007 February 21-27 and 2007 May 2-8. The SITe CCD has a field of view of $26 \mathrm{arcmin}^{2}$ at the $f / 16$ Cassegrain focus of the $1 \mathrm{~m}$ telescope. Of the $30-40$ other stars recorded per field, several bright stars were used as local differential standards. 

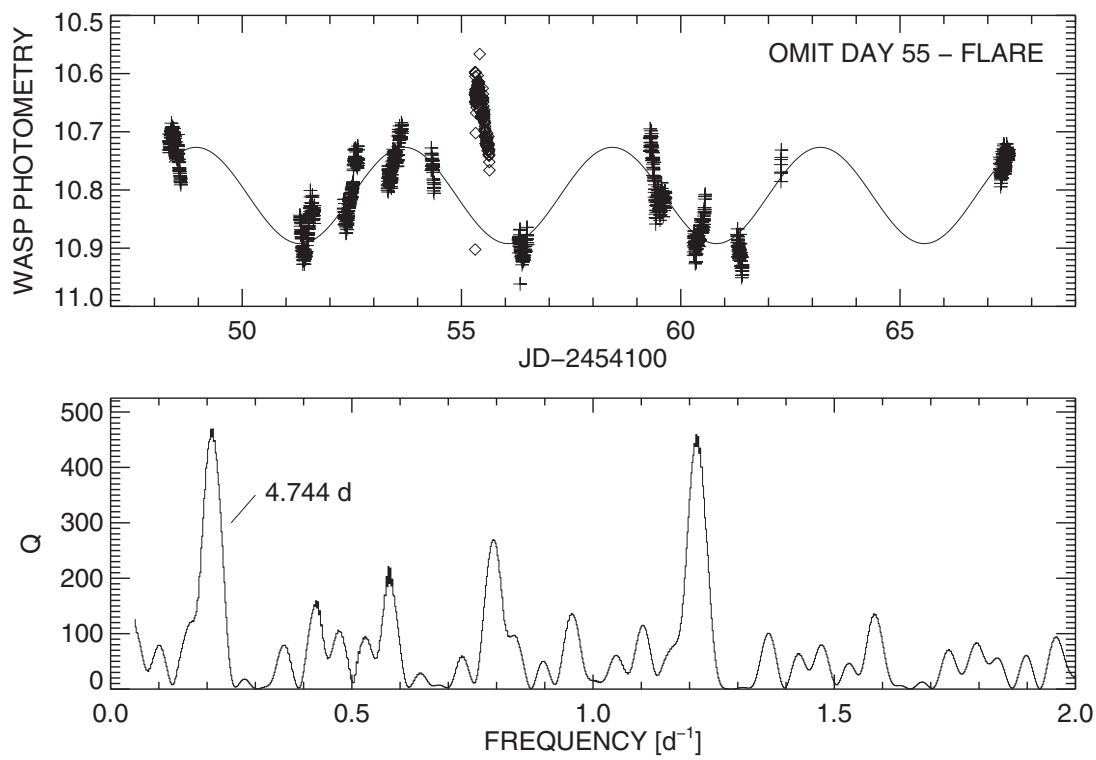

Figure 2. Upper panel: light curve of TW Hya from WASP-S measured typically every $120 \mathrm{~s}$. Errors in the magnitude per bin range from 3 to $5 \mathrm{mmag}$. A substantial flare occurred during JD 2454100+55 (points denoted by open diamond symbols) and this night has been omitted from the period search. A sine curve with the derived period (see below) overlays the data. Lower panel: the periodogram reveals one strong period during this time at a frequency of $0.210800\left(\right.$ day ${ }^{-1}$ ) corresponding to 4.744 days. The peak at 1.21 day $^{-1}$ corresponds to an alias of +1 cycle day $^{-1}$.

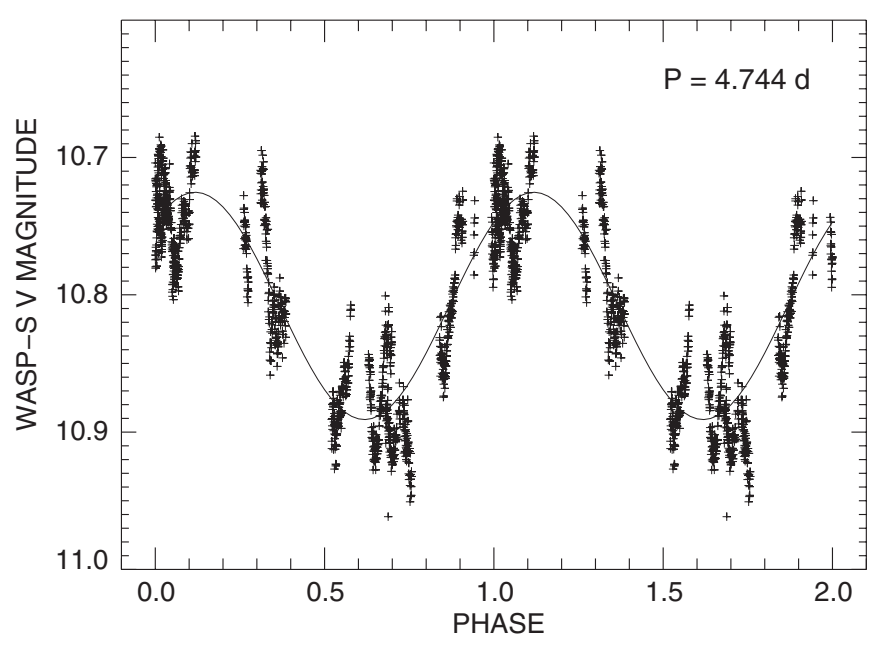

Figure 3. WASP-S photometry phased to the period of 4.744 days where the flaring episode identified in Figure 2 has been omitted. The data are repeated twice in this figure. These magnitudes should be better than $1 \%$ for a star as bright as TW Hya. The substantial real short-term variability is similar to that found in other T Tauri stars.

The observations were made differentially as this allowed useful data to be obtained under conditions that were not always photometric. The methods used to produce these photometric observations are discussed in detail by Lawson et al. (2001) and Lawson \& Crause (2005). The SAAO results are shown in Figure 4(panel C) along with the WASP-S photometry (panel B), and the Chandra first-order flux (panel A, also see discussion in the following section). The flaring episode during day 55 found in the WASP-S data can also be seen in the SAAO photometry. Additionally, the X-ray flare event near day 58.5 in Figure 4 appears related to an optical flare about 20 minutes later during which the SAAO photometry shows a brightening of $\sim 0.1 \mathrm{mag}$. Outside of the flaring episodes, the SAAO photometry is consistent with the 4.74 day period.
Photometric data in $V$ band are available from the All Sky Automated Survey (ASAS) of the University of Warsaw (Pojmanski 2002) located at the Las Campanas Observatory of the Carnegie Institution of Washington. These fully automated photometric monitoring measurements are publicly available from http://www.astrouw.edu.pl/asas/. They are shown in the panel $\mathrm{D}$ of Figure 4 where the variation is in harmony with the 4.74 day period identified in the WASP-S data, although the cadence of ASAS ranges from 2 to 3 days. Of particular interest is the high point near day 57.6, which appears to signal a brightening event contemporaneous with the increase in the Chandra accretion-line flux (see Section 6).

Thus, photometric variability results from a variety of physical processes in these accreting systems, including flaring episodes superposed on a light modulation of varying periods. These results illustrate how challenging it is to seek a photometric period for TW Hya. We adopt the photometric period of 4.744 days as the period of reference for these observations. None of our results depend on the observed period.

\section{CHANDRA X-RAY OBSERVATIONS}

HETG spectra were obtained during four separate Chandra pointings comprising a Large Observing Project that totaled $500 \mathrm{ks}$ and spanned the interval between 2007 February 15 and 2007 March 3 (Table 1). These spectra, their plasma diagnostics, and the model implied by the X-ray observations are discussed in detail in Brickhouse et al. (2010). These authors emphasized the importance of recognizing that the Chandra first-order $\mathrm{X}$-ray flux includes both emission lines and continua arising in different parts of the TW Hya system. The total first-order flux (spanning 2-27.5 $\AA$ ) is dominated by continuum emission produced by the stellar coronal plasma at $\sim 10^{7} \mathrm{~K}$. In addition, the spectrum allows the separation of emission lines according to their origin. The high-temperature $\left(1.2 \times 10^{7} \mathrm{~K}\right)$ emission lines of $\mathrm{Mg}$ XII, Si XIII, and Si XIV arise in the corona. The lower temperature $\left(2.5 \times 10^{6} \mathrm{~K}\right)$ emission lines (N VII, O VIII, 

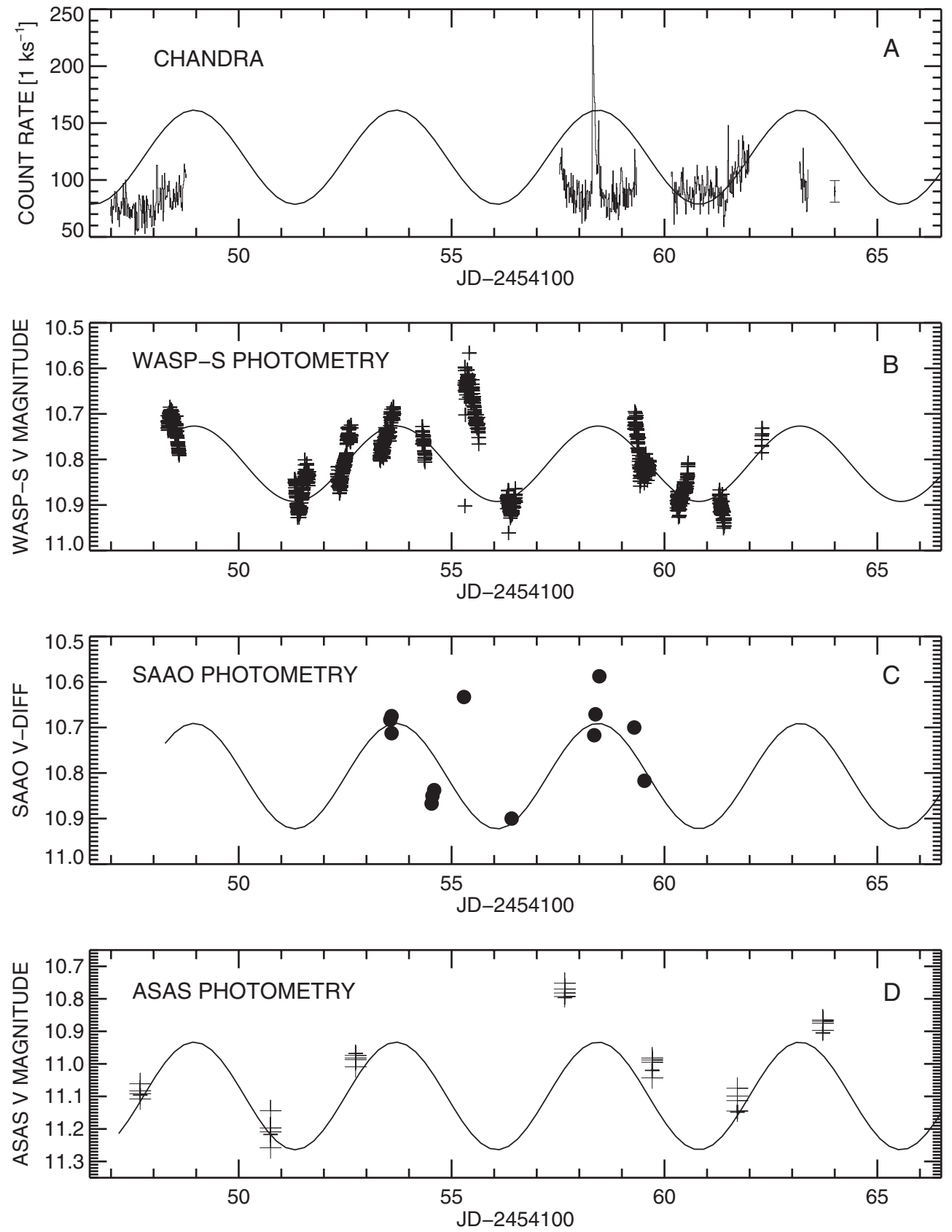

Figure 4. Chandra first-order (coronal) X-ray light curve binned to $1 \mathrm{ks}$ (panel A) and contemporaneous photometry: WASP-S (panel B), SAAO $V$-band differential photometry (panel C), and ASAS photometry from five apertures (panel D). A sine curve with the 4.74 day period derived from WASP-S photometry with arbitrary amplitude overlays the Chandra flux and the SAAO and ASAS photometry. Two white-light flaring episodes appear as indicated by the photometry during day 55 and days 57-58; the latter coincides with the Chandra (coronal) X-ray flare.

Table 2

Ground-based Observations

\begin{tabular}{lccccc}
\hline \hline $\begin{array}{l}\text { Observatory } \\
\text { Telescope }\end{array}$ & Instrument & Data & Observer & $\begin{array}{c}\text { JD Start } \\
(-2454100)\end{array}$ & $\begin{array}{c}\text { JD End } \\
(-2454100)\end{array}$ \\
\hline WASP-S & & Photometry & Robotic & 48.33 & 67.48 \\
TNG & SARG & Spectra & DDT & 48.57 & 49.60 \\
SAAO & Cassegrain & Spectra & Crause/Lawson & 53.35 & 59.53 \\
SAAO & & Photometry & Crause/Lawson & 53.56 & 59.53 \\
ASAS & & Photometry & Robotic & 47.69 & 63.72 \\
VBO & Echelle & Spectra & Mallik & 59.25 & 63.48 \\
Magellan/Clay & MIKE & Spectra & Dupree & 57.51 & 59.93 \\
Magellan/Clay & MIKE & Spectra & Bonanos & 60.68 & 60.69 \\
Pico dos Dias & Coudé & Spectra & Luna & 60.68 & 61.77 \\
Gemini-S & PHOENIX & Spectra & Schuler & 60.71 & 60.74 \\
SSO & Echelle & Spectra & Bessell/Lawson & 61.12 & 66.02 \\
\hline
\end{tabular}



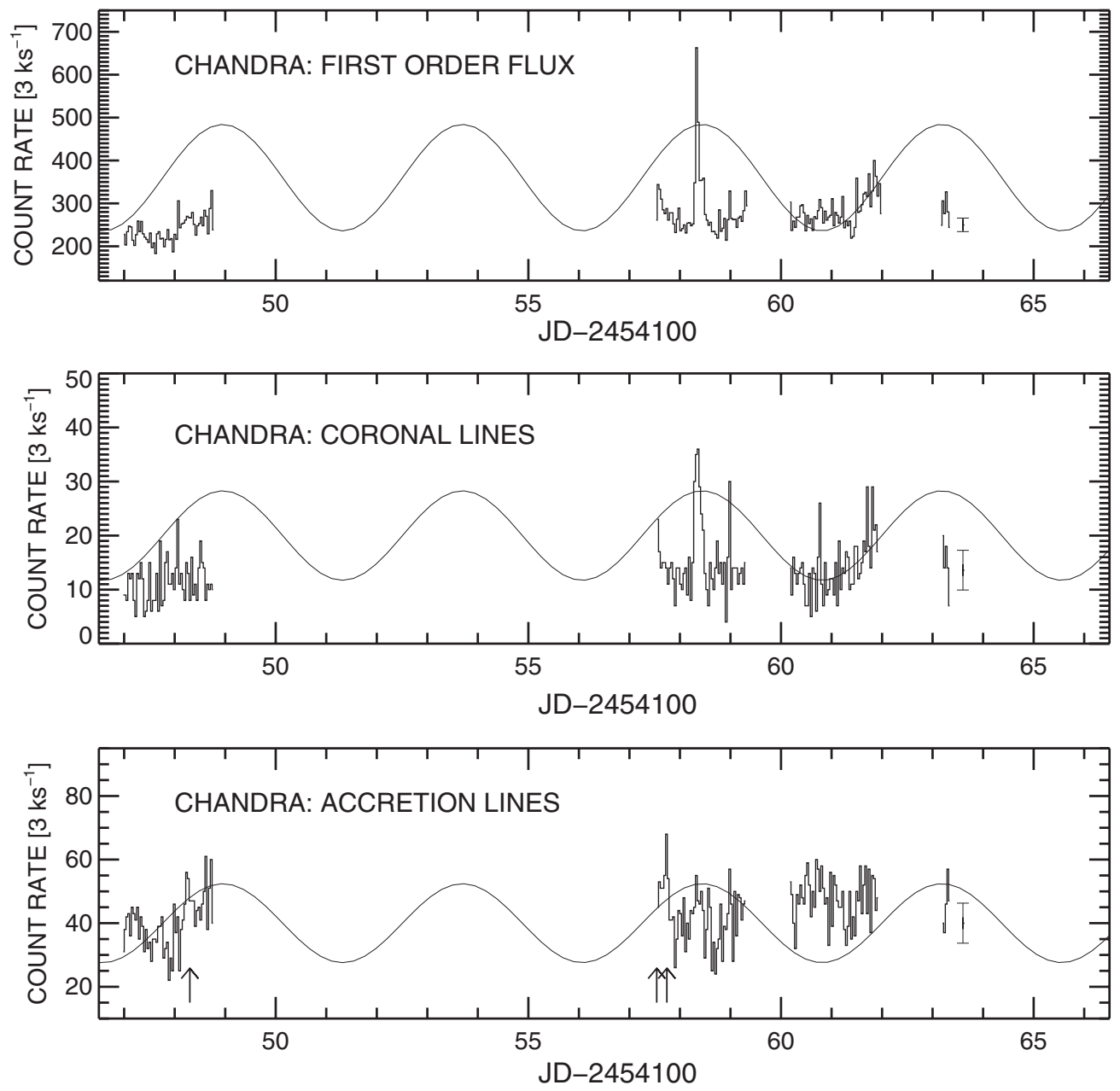

Figure 5. Chandra first-order light curve (top panel) containing lines and continuum and a separation of the emission line X-ray spectrum into features arising from the hot stellar corona (middle panel) and the accretion shock (lower panel). All data are binned over $3 \mathrm{ks}$. A sine curve with the 4.74 day period derived from contemporaneous photometry, and arbitrary amplitude is overlaid in each panel to aid the eye. It is easy to see from the coronal lines (middle panel) that the X-ray flare (top panel) during day 58 is predominantly a coronal event as found by Brickhouse et al. (2010). The accretion lines vary in flux by a factor of 1.5 , and two localized "accretion events" (marked by arrows in the lower panel) occur at the same photometric phase.

Ne IX, Fe XVII, and $\mathrm{Mg} \mathrm{XI}$ ) form in the accretion shock as defined in the model of Brickhouse et al. (2010). These will be referred to as X-ray accretion lines. The newly identified large post-shock coronal region produces the $\mathrm{O}$ VII emission. For comparisons with the optical spectra and photometry in the following sections, we use either the total first-order flux (representing the stellar corona) or a selection of emission lines originating either in the accretion shock or the corona as defined above.

No periodicity in the first-order X-ray flux can be found from analysis of the totality of observations. Comparison of the optical photometric period of 4.74 days to the X-ray flux (Figure 4) shows modulation during the third Chandra segment on days 60-62 in agreement with photometry. However the preceding and following segments do not follow the photometric curve and the agreement on days 60-62 may be coincidental. The first Chandra segment appears anomalously low when compared to the photometric period, unless there is a deviation from the average coronal flux during this segment.

A lack of correlation may not be surprising since the optical light giving the photometric period consists of variable continuum emission from the star itself, the hot accretion regions (spots/rings), the activity from plages and active regions, flaring episodes, and possibly a contribution from the accretion disk. In any case, the total coronal emission is not correlated with the optical photometric period during these observations. This lack of X-ray periodicity is consistent with the results of Stassun et al. $(2006,2007)$ from the young Orion Nebula Cluster where $95 \%$ of the large COUP sample exhibited no link between X-ray variability and optical photometry.

The X-ray spectrum of TW Hya offers additional insight (Brickhouse et al. 2010). Figure 5 includes the Chandra total flux (top panel), the emission line flux from the corona (middle panel), and the accretion shock (lower panel). The corona is principally responsible for the flare during day 58 , as well as the slow increase in flux during day 61. Enhancement episodes observed in the accretion emission lines occurred near days 48 and 57 (marked by arrows in Figure 5). Separating out these components, we find that accretion may contribute slightly to the total X-ray variability. Accretion appears to cause the brightening of the total flux during the first Chandra interval (Day 48.2) as well as at the start of the second Chandra segment (Day 57.5).

We find that variation in the total $X$-ray flux arises from both accretion and coronal variability and neither the accretion nor coronal components exhibits the periodicity of the optical 
photometry. Flaring episodes in the corona can be identified with some brightenings found in the optical photometry. In the following sections, the X-ray variations are related to the optical line profiles.

We emphasize that the observed X-ray accretion-line flux represents a complicated mix of temperature, column density in the accreting material, and the electron density. The accretion rate could increase, due to enhancement of the accretion column density, which for a fixed filling factor, absorbs and hence reduces the observed soft X-ray line flux. Or, the accretion rate could also increase creating a larger filling factor, accompanied by a decrease in absorbing column density, and an increased soft X-ray line flux. In Section 6, we explore the optical spectroscopic response to the observed increased accretion-line flux.

\section{OPTICAL AND INFRARED SPECTROSCOPIC OBSERVATIONS}

Spectra were obtained from seven observatories spanning the Chandra pointings in order to follow the variation in the strength and appearance of the emission profile, to search for the relationship between the accretion process, the stellar atmosphere and the stellar wind, and to relate any line variation to the X-ray flux from the corona and the accretion shock. We summarize the telescopes and instruments in the following sections (also Table 2), and then present an examination of line profiles in the following sections.

\subsection{Telescopio Nationale Galileo (TNG)_Canary Islands}

Telescope time on the $3.58 \mathrm{~m}$ Telescopio Nationale Galileo (TNG) was kindly made available through the Director's Discretionary program to coincide with the first Chandra segment during the nights of 2007 February 17-18. Unfortunately, one night was clouded out. The SARG, a high-efficiency echelle spectrograph with a mosaic of two $2 \times 4 \mathrm{~K} \mathrm{CCD}$ detectors, was binned at $2 \times 2$ pixels. Aperture 2 was selected, with a slit size of $0.8 \times 5.3$ arcsec giving a spectral resolution of $\sim 57,000$. Luca DeFabrizio who acquired the data in service mode kindly reduced the spectra using modified IRAF ${ }^{12}$ software.

\subsection{Vainu Варри Observatory (VBO)—India}

The 2.3 m telescope of Vainu Bappu Observatory (VBO) in Kavalur, India made observations of TW Hya for five nights from 2007 February 27 to March 3. Exposure times of $2700 \mathrm{~s}$ were used for all spectra. The fiber-fed echelle spectrograph provided a resolution of 28,000. No sky subtraction was applied. The spectra were reduced with IRAF, and continuum normalization excluded the $\mathrm{H} \alpha$ emission. A preliminary report of these observations is given in Mallik et al. (2010).

\subsection{South Africa Astronomical Observatory (SAAO)_South Africa}

Medium-resolution spectroscopy of TW Hya was obtained with the $1.9 \mathrm{~m}$ telescope at the Sutherland field station of the SAAO between 2007 February 21 and 28. The Cassegrain spectrograph was used with grating " 5 " that has a 2 pixel resolution at $\mathrm{H} \alpha$ of $1 \AA(\lambda / \Delta \lambda \sim 6000)$. Exposure times ranged between 90 and $1800 \mathrm{~s}$ in order not to saturate the peak of the $\mathrm{H} \alpha$ emission line profile, or to obtain higher signal-to-noise ratio $(\mathrm{S} / \mathrm{N})$ measurements of the $\mathrm{H} \alpha$ line wings and surrounding continuum. The spectra were reduced with IRAF. ${ }^{12}$ The S/N ranged from 50 to 100 in the continuum.

\subsection{Mt. Stromlo and Siding Spring Observatory (MSSO)_Australia}

Spectroscopy of TW Hya was obtained with the $2.3 \mathrm{~m}$ telescope and echelle spectrograph at Siding Spring Observatory (SSO) between 2007 March 1 and 6. The SSO spectra have a 2 pixel resolution of $0.4 \AA$ at $\mathrm{H} \alpha(\lambda / \Delta \lambda \sim 16,400)$. Exposure times of 600-1200 s yielded spectra with $S / N$ of $50-125$ in the nearby continuum.

\subsection{Las Campanas Observatory (Magellan:Clay)—Chile}

The MIKE double-echelle spectrograph (Bernstein et al. 2003) mounted on the $6.5 \mathrm{~m}$ Magellan/Clay telescope at Las Campanas Observatory, Chile, was dedicated for three consecutive nights (2007 February 26-28) to obtain several hundred echelle spectra spanning the optical region. An additional spectrum was obtained on 2007 March 1. A slit of $0.75 \times 5$ arcs was used yielding a 2 pixel resolution of $\Delta \lambda / \lambda \sim 26,000$ on the blue side $(\lambda \lambda 3350-5000)$ and $\sim 36,000$ on the red side ( $\lambda \lambda 4900-9300)$. Spectra were taken with short exposures ranging from 45 to $90 \mathrm{~s}$ (to avoid saturation of the $\mathrm{H} \alpha$ emission) mixed with longer exposures (360-600 s) to detect weaker features. The IDL pipeline developed by S. Burles, R. Bernstein, and J. S. Prochaska ${ }^{13}$ was used to extract the spectra, and IRAF software was used for analysis.

\subsection{Pico dos Dias Observatory-Brazil}

Optical spectra were obtained at Observatorio Pico dos Dias, Brazil, during the nights from February 26 through 2007 March 1. The Coudé spectrograph attached to the $1.6 \mathrm{~m}$ telescope was used with the WI098 CCD and the $600 \ell \mathrm{mm}^{-1}$ grating, resulting in a resolution of $\lambda / \Delta \lambda \sim 13,000$ at $6500 \AA$. The average exposure time was $1800 \mathrm{~s}$.

\subsection{Gemini South-Chile}

The PHOENIX IR spectrograph on the $8 \mathrm{~m}$ Gemini-South (Gemini-S) telescope was used to record the spectrum of the He I $10830 \AA$ line on 2007 March 1 with a 4 pixel slit giving a resolution of $\lambda / \Delta \lambda \sim 50,000$. Two exposures of $900 \mathrm{~s}$ each were nodded by 4 arcsec on the CCD. Flat fielding of the exposures was performed with IRAF routines. The known dispersion of PHOENIX at this grating position was applied to the spectra and they were aligned to the Mg I line at $10811.11 \AA$.

\section{THE $\mathrm{H} \alpha$ AND $\mathrm{H} \beta$ LINES}

The $\mathrm{H} \alpha$ line emission dominates the optical spectrum of TW Hya and its enhanced width is generally believed to signal the presence of accretion in young stars (Bertout 1989; Hartmann et al. 1994; Alencar \& Batalha 2002). In this section, nightto-night variability in the line strength is first addressed from the sequence of observations spanning 19 days, followed by a discussion of the line profiles of $\mathrm{H} \alpha$ and $\mathrm{H} \beta$.

\footnotetext{
13 See http://web.mit.edu/ burles/www/MIKE/.
} 


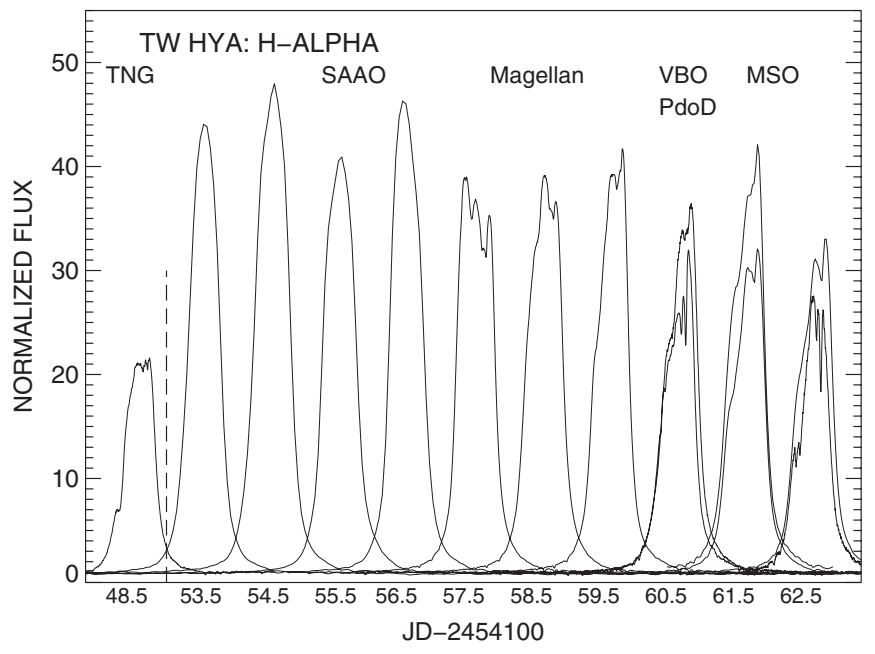

Figure 6. Nightly averages of $\mathrm{H} \alpha$ profiles. The spectroscopic resolution and the number of spectra per night both vary substantially. The lower resolution profiles from SAAO (Days 53.5-56.5) have been multiplied by 0.5 to normalize them to the Magellan spectra taken at the same time on day 58.5. Note the gap in the $X$-axis between the first and second profile (marked by a broken line). The facilities used are marked.

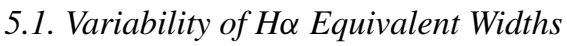

All $\mathrm{H} \alpha$ spectra were reduced to a heliocentric scale and normalized to the neighboring continuum using the IRAF tool continuum generally with a three- or fifth-order Legendre function. The region near the $\mathrm{H} \alpha$ emission line was omitted from the continuum fit. Averages of the profiles are shown in Figure 6 where high emission during four consecutive days is followed by lowered emission. Equivalent widths were obtained by integrating the continuum-normalized spectra over a $25 \AA$ region from 6550 to $6575 \AA$. Comparison between the X-ray accretion-line fluxes, the $\mathrm{H} \alpha$ equivalent widths, and the optical photometric period is shown in Figure 7. Values from the spectra of the six observatories are shown separately because the spectral resolution differs among the instruments.

Inspection of the $\mathrm{H} \alpha$ equivalent widths shows that they do not correlate well with the optical photometric period. While brief sequences of agreement might occur (for instance SAAO (Days 54-56), Magellan (Days 57-59), or MSSO (Days 61-65)), none of the six spectral sets of $\mathrm{H} \alpha$ measures is totally consistent with the photometric period determined over the same time interval. We sought a periodicity in the SAAO spectra which has the longest time span, but no meaningful period exists in the data.

The underlying assumption in the interpretation of the $\mathrm{H} \alpha$ profiles holds that the emission arises from the accreting material as it is channeled along magnetic field lines from the disk to the star (Muzerolle et al. 2001; Natta et al. 2004), and fitting the profile with accretion models gives an estimate of the mass accretion rate. Thus we might expect the equivalent width of $\mathrm{H} \alpha$ to exhibit behavior similar to that of the X-ray accretion lines. Inspection of Figure 7, in particular, during the second and third Chandra pointings beginning day 57 does not show a convincing correlation. The flux of X-ray accretion lines decreases from day 57.5 to day 58.5, and the spectra from SAAO and Magellan do show a decrease in the $\mathrm{H} \alpha$ flux. However, beginning at day 60.2, the accretion lines are at a high level again (comparable to day 57.5). While the Vainu Bappu spectra indicate a decrease in the equivalent width of $\mathrm{H} \alpha$ between day 60.2 and day 61.3 similar to the change in the X-ray accretion-line flux, the Pico dos Dias spectra show a decreasing $\mathrm{H} \alpha$ flux between day 60.7 and day 61.6 when the X-ray accretion fluxes have not changed. We find no correlation during the simultaneous observations (Days 57.5-61.9) among the Ho equivalent widths, the flux of $X$-ray accretion lines, and the optical photometric period. Study of the line profiles themselves in the following section is more revealing.

\section{2. $H \alpha$ and $H \beta$ Profile Changes}

For three continuous nights (Days 57.51-59.92), MIKE spectra from Magellan/Clay with echelle resolution were obtained with high frequency (about nine exposures of $100 \mathrm{~s}$ or less per hour in addition to hourly longer exposures). An additional spectrum was taken on the fourth night.

Line profiles by their asymmetries can signal the presence of differential mass motions in the line-forming regions of optically thick lines (Hummer \& Rybicki 1968). In a static atmosphere, a line absorption coefficient remains at rest centered on the profile. However, with differential expansion, the absorption coefficient moves to shorter wavelengths, weakening the short-wavelength side of the profile, and creating a "blue" side that is weaker than the "red" side-a red asymmetry. And the opposite occurs when differential inflow is present. Thus the simple profile shape of these lines reveals the atmospheric dynamics in the lineforming region. We seek such signatures of asymmetry in the line profiles.

The first night of the MIKE observations (Days 57.51-57.92) exhibited an unusual $\mathrm{H} \alpha$ profile (Figure 8, left panel) in that it has a blue asymmetry (negative velocity peak is stronger than the positive velocity peak) indicating inflowing material. The $\mathrm{H} \beta$ line (Figure 8, right panel), which is less optically thick than $\mathrm{H} \alpha$ is striking in the inflow signature of blue asymmetry and a substantial absorption feature near $+35 \mathrm{~km} \mathrm{~s}^{-1}$. $\mathrm{H} \beta$ occurs on both the red and blue sides of MIKE and we have selected the profile from the blue arm of the MIKE spectrograph since the line is positioned near the center of the echelle order providing good photon statistics. Exposures for the blue side are generally longer (100-360 s) than for the red side which must be shorter so as not to saturate the $\mathrm{H} \alpha$ emission line. The $\mathrm{H} \beta$ profile clearly shows a signature of inflowing material with its broad absorption near $+35 \mathrm{~km} \mathrm{~s}^{-1}$ that may correspond also to a feature in the $\mathrm{H} \alpha$ profile. ${ }^{14}$ Over the subsequent three nights of observation, the short-wavelength wings of both $\mathrm{H} \alpha$ and $\mathrm{H} \beta$ become systematically weaker.

The $\mathrm{H} \alpha$ profile during Night 1 (Days 57.51-57.92) of the MIKE observations is unusual and not seen in the $\mathrm{H} \alpha$ profiles that we have assembled intermittently over seven years (2003-2010). This profile is broad, and relatively "flat-topped" although the short-wavelength peak is stronger than the longwavelength peak (blue asymmetry). Alencar \& Batalha (2002) in their study of $42 \mathrm{H} \alpha$ profiles spanning two years consistently report an asymmetric profile, much like Nights 2,3 , and 4 reported here (Figure 8). Straightforward interpretation of the $\mathrm{H} \alpha$ profile suggests that the Night 1 profile indicates inflow (i.e., material moving toward the star) and a stellar wind that is absent or extremely weak, only to become re-established during the following nights. The profile of $\mathrm{H} \beta$ confirms this interpretation as it exhibits a strong inflow signature, and similarly increasing wind absorption each successive night.

The Night $1 \mathrm{H} \alpha$ profiles in Figure 8 appear to represent the intrinsic stellar/accretion emission profile unmodified by

\footnotetext{
14 The $\mathrm{H} \alpha$ region has several water vapor lines which are discussed in the Appendix. They do not affect our conclusions.
} 


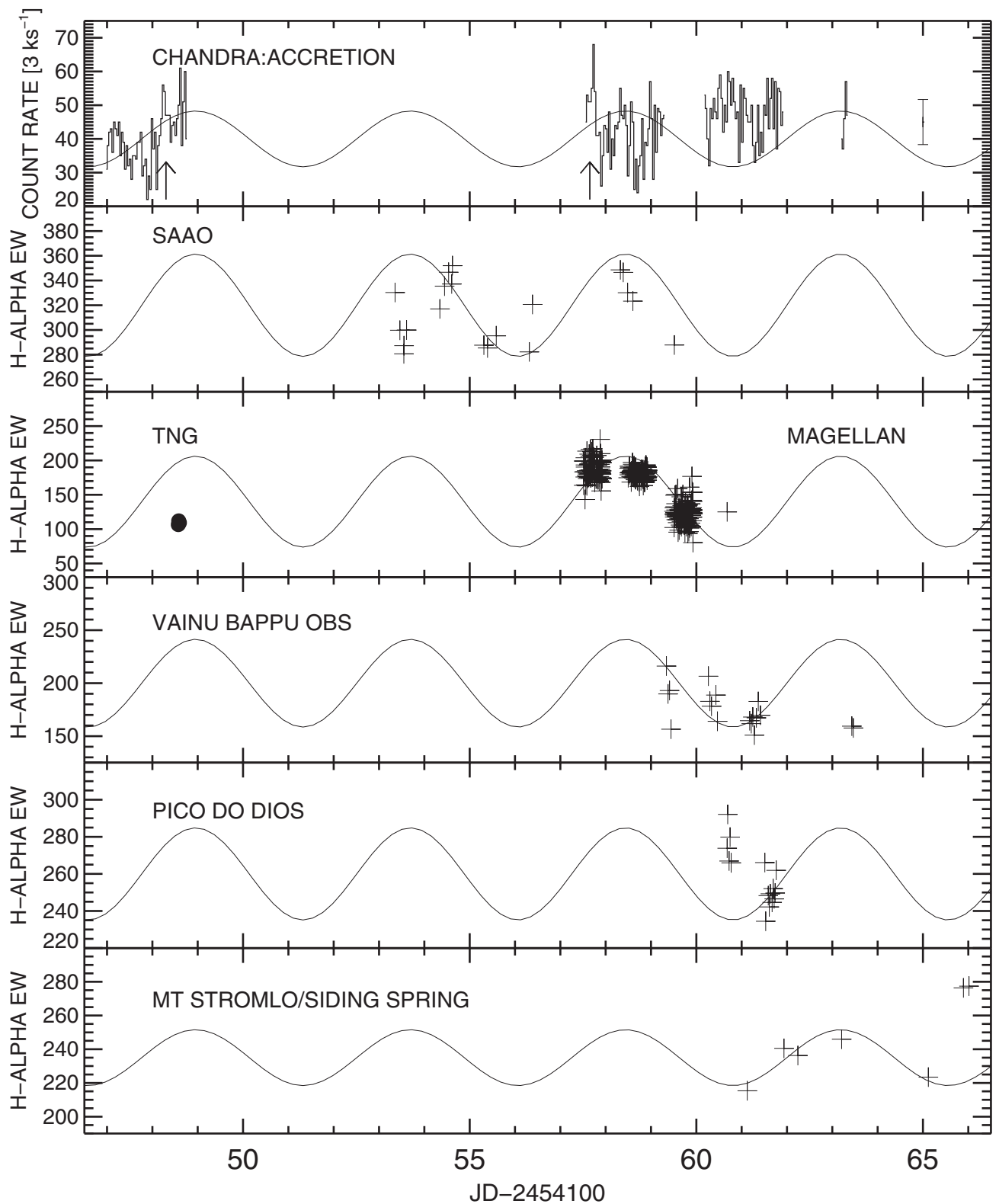

Figure 7. Chandra accretion-line flux binned to $3 \mathrm{ks}$ and $\mathrm{H} \alpha$ equivalent widths for all of the spectroscopic observations. A sine curve with the period of 4.744 days from WASP-S photometry and arbitrary amplitude is overlaid, and placed to guide the eye as to the H $\alpha$ strength.

a stellar wind. The red (long wavelength) and blue (short wavelength) emission peaks are clearly correlated as shown in Figure 9. This indicates that a single process produces the emission line and suggests that the intrinsic $\mathrm{H} \alpha$ line profile is approximately symmetric and broad. During subsequent nights, absorption from outflowing material sets in. This systematic and uniform decrease of the short-wavelength flux in $\mathrm{H} \alpha$ is shown in Figure 10 where the ratio of normalized fluxes from a $1 \AA$ bandpass on the short-wavelength side (blue) to the longwavelength side (red) is given for three nights. During this time, the $\mathrm{H} \beta$ line behaves similarly. The positive velocity component in $\mathrm{H} \beta$ remains constant while the negative velocity side of the $\mathrm{H} \beta$ line becomes systematically weaker as the wind also appears in the less optically thick line. The abrupt change of the $\mathrm{H} \alpha$ blue:red ratio during Night 1 is associated with an accretion event (Section 6 following) and the profile becomes modified as the wind from the star develops absorbing the hydrogen emission.
The absorption on the short-wavelength side of the $\mathrm{H} \alpha$ line yields an estimate of the optical depth in the neutral component of the stellar wind. At a velocity of $-100 \mathrm{~km} \mathrm{~s}^{-1}$ in the $\mathrm{H} \alpha$ profile, the wind increases to an optical depth of $\sim 0.5$ by the third night, assuming no wind absorption on the first night. A lower limit to the column density of hydrogen required to produce this absorption can be found with the assumption that $T=10^{4} \mathrm{~K}$ in a wind that is predominantly neutral hydrogen, and by taking the line absorption coefficient to be thermally broadened. These assumptions yield a lower limit of, $N_{H} \times L>3 \times 10^{19} \mathrm{~cm}^{-2}$ in the wind from the polar regions of TW Hya. This column density is enhanced in relation to a quiet-Sun coronal value of $\sim 2 \times 10^{19} \mathrm{~cm}^{-2}$.

The source of the extremely broad $\mathrm{H} \alpha$ and $\mathrm{H} \beta$ profiles has been traditionally associated with the pre-shock accreting material. Early models (Muzerolle et al. 1998a) attributed a redshifted $\mathrm{H} \alpha$ profile to its origin in the magnetically confined accretion stream resulting in narrow $\mathrm{H} \alpha$ emission. Later models 

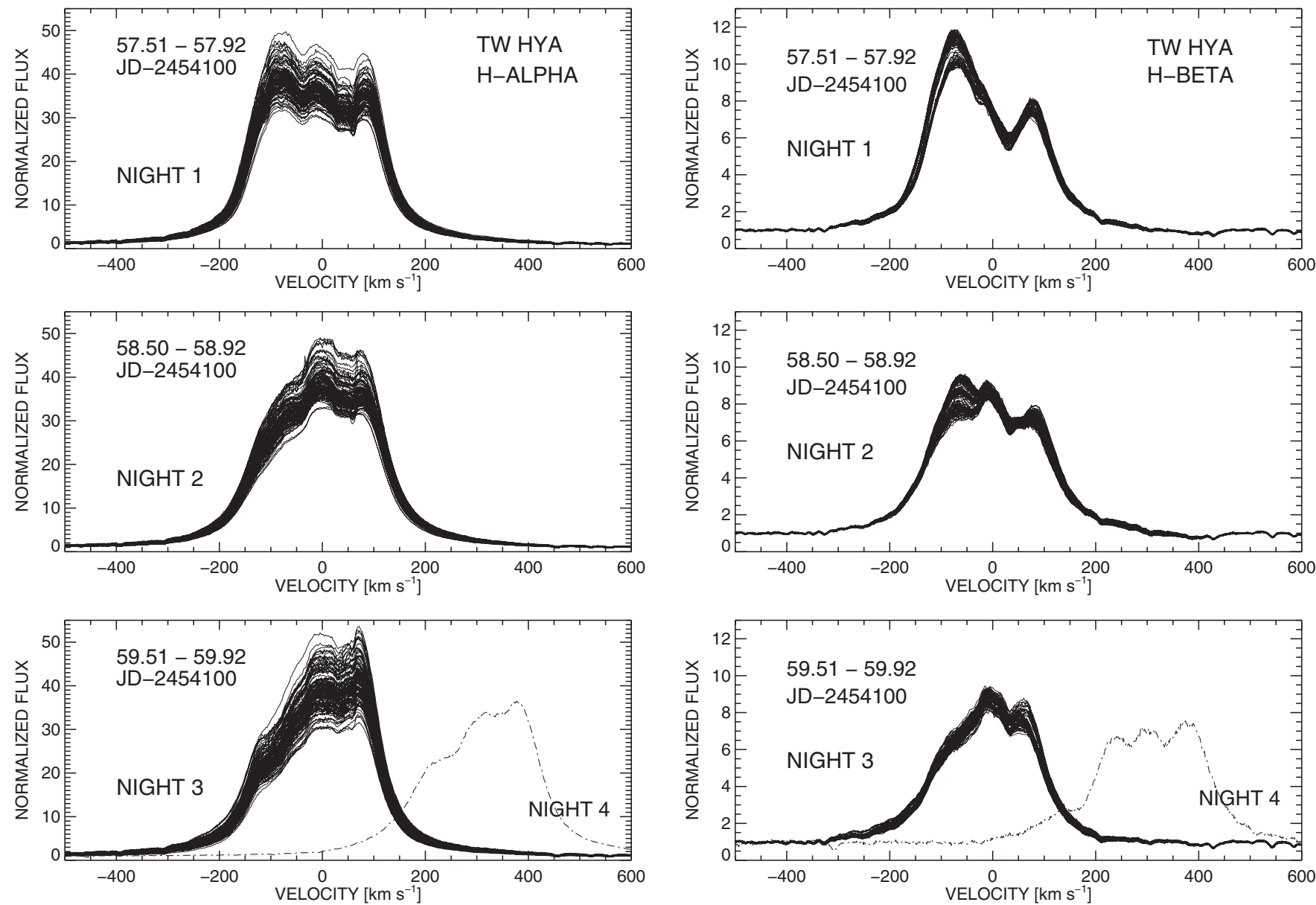

Figure 8. Four consecutive nights of continuous observations of the $\mathrm{H} \alpha$ (left panel) and $\mathrm{H} \beta$ (right panel) profiles from Magellan reveal substantial systematic changes principally on the short-wavelength side of the emission profile. The profile from Night 4 is offset by $300 \mathrm{~km} \mathrm{~s}^{-1}$ for display.

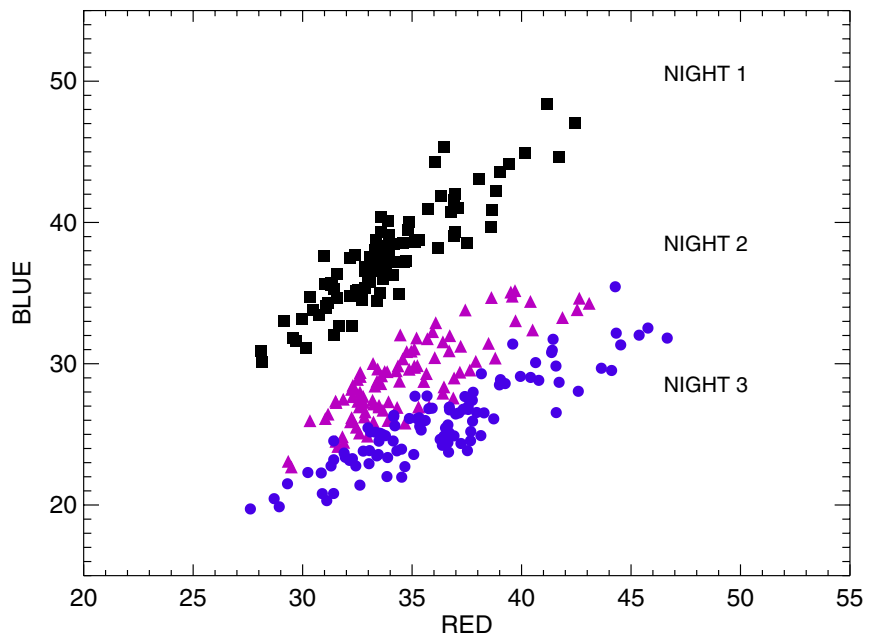

Figure 9. Strength of the blue wing in the $\mathrm{H} \alpha$ emission line as a function of the strength of the red wing from three sequential nights of Magellan spectra: days 57-59. Both sides of the line are correlated indicating that a single process produces this intrinsically broad line. Filled squares $(\mathbf{\square})$ correspond to Night 1 (starting JD 2454157.5); filled triangles ( $\mathbf{\Delta}$ ) correspond to Night 2 (JD 2454158.5); filled circles (•) mark Night 3 (JD 2454159.5). Fluxes are measured in a $1 \AA$ wide band positioned $\pm 2 \AA$ from line center in continuumnormalized spectra. The decreasing slope over the three nights results from the increasing wind opacity that weakens the "blue" side of the line profile.

(A color version of this figure is available in the online journal.)

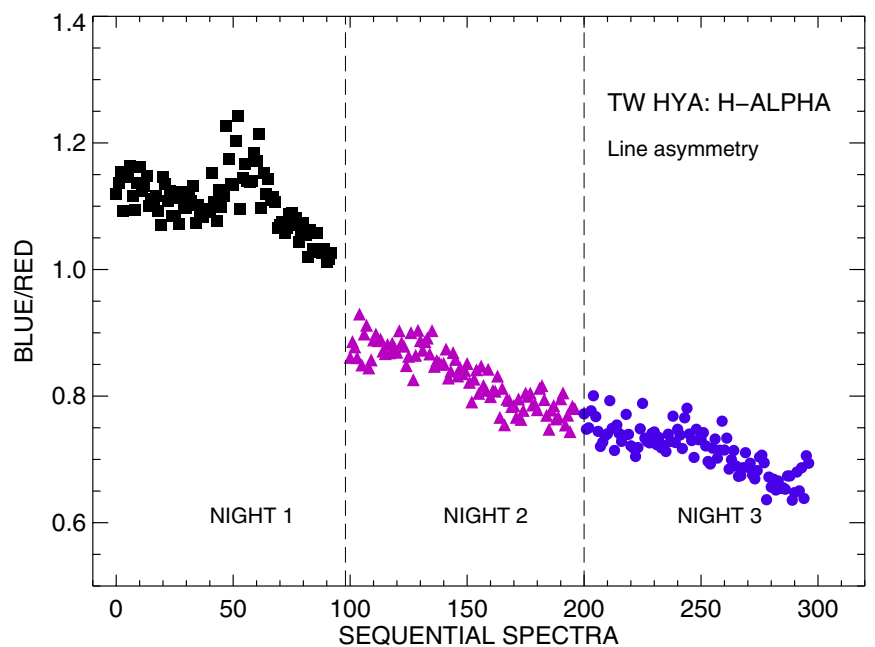

Figure 10. Blue:red asymmetry as a function of time over three consecutive nights. The "blue" and "red" fluxes are taken from a $1 \AA$ wide band positioned $\pm 2 \AA$ from line center in continuum-normalized spectra. A systematic decrease of the ratio occurs over three successive nights following generally higher activity during the first night after the accretion event (corresponding to spectrum $\sim 50$ ) described in Section 5.2.

(A color version of this figure is available in the online journal.) 


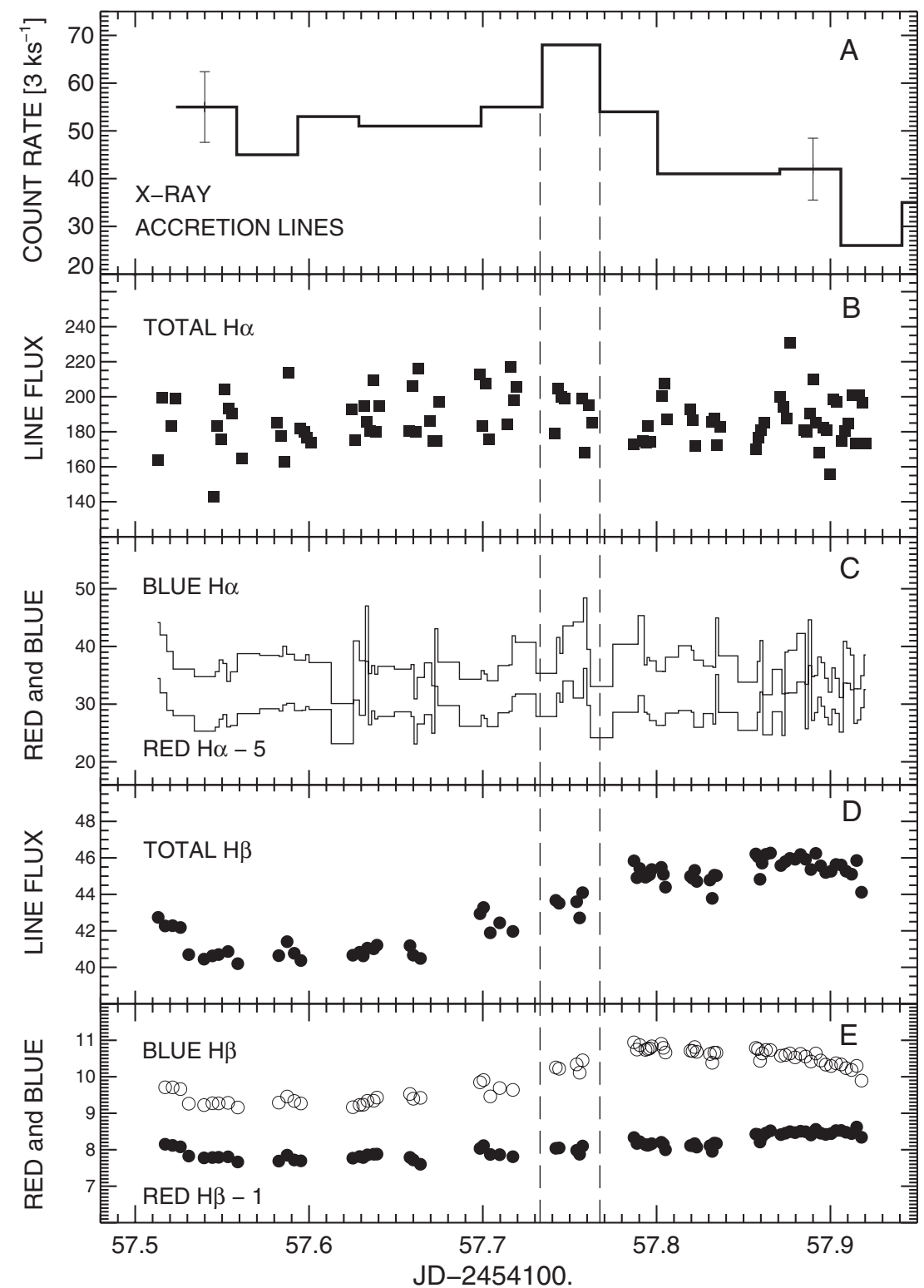

Figure 11. $\mathrm{H} \alpha$ and $\mathrm{H} \beta$ fluxes during the accretion event of day 57.74. Also included are the fluxes from a 1 A region on the short (blue) and long (red) wavelength sides of each line. The red $\mathrm{H} \alpha$ flux has been lowered by 5 units in this figure; similarly the red $\mathrm{H} \beta$ flux has been lowered by 1 unit for display.

(Muzerolle et al. 2001; Kurosawa et al. 2011) incorporated line broadening and multidimensional non-LTE radiative transfer in the accretion stream, but the profiles remain pointed and not similar to the profile of TW Hya during Night 1, which is distinctly broader and flatter than typical profiles for classical $\mathrm{T}$ Tauri stars spanning a wide variation in accretion activity (cf. Muzerolle et al. 2001; Figure 11). Since the polar regions of TW Hya are facing us, redshifted emission with velocities up to $\sim+500 \mathrm{~km} \mathrm{~s}^{-1}$ (the expected ballistic free-fall velocity assumed for the pre-shock material) would be expected if the emission was produced by the infalling accretion stream. But this $\mathrm{H} \alpha$ profile is broad indicating plasma with velocities both approaching and receding up to $\pm 150 \mathrm{~km} \mathrm{~s}^{-1}$. Whereas a preshock accretion stream might account for redshifted emission, it cannot explain the blueshifted emission considering that TW Hya faces us pole-on. The FWHM of the flat-topped $\mathrm{H} \alpha$ profile during Night 1 ranges from 250 to $300 \mathrm{~km} \mathrm{~s}^{-1}$; the thermal width of hydrogen at $10^{4} \mathrm{~K}$ is only $21 \mathrm{~km} \mathrm{~s}^{-1}$. Clearly, the large values of the line width are characteristic of supersonic turbulence in the region where $\mathrm{H} \alpha$ is formed. The profile requires the presence of a substantial turbulent component of $250-300 \mathrm{~km} \mathrm{~s}^{-1}$ to produce such broadening. Note that the width of the turbulent component of the Ne Ix lines formed in the accretion shock is also high, at $165 \pm 18 \mathrm{~km} \mathrm{~s}^{-1}$ (Brickhouse et al. 2010). Correlated strengths of the blue and red side of the Ho line and the enhanced $H \alpha$ widths suggest that this emission (as well as $H \beta$ ) is associated with the subsequent cooling from the accretion shock and not from the accretion flow channeled from the circumstellar disk to the star. The behavior of these spectroscopic diagnostics with time discussed in the following section can inform this model as well.

Studies of accreting $\mathrm{T}$ Tauri stars suggest that the width of the line at the $10 \%$ level is related to the accretion rate (White \& Basri 2003; Natta et al. 2004; Curran et al. 2011). Here, the width of the $\mathrm{H} \alpha$ line at the $10 \%$ level above a continuum-subtracted normalized profile varies between 390 and $465 \mathrm{~km} \mathrm{~s}^{-1}$, values that translate into an accretion rate from $9.0 \times 10^{-10}$ to $4.2 \times$ $10^{-9} M_{\odot} \mathrm{yr}^{-1}$ according to the relationship proposed by Natta 


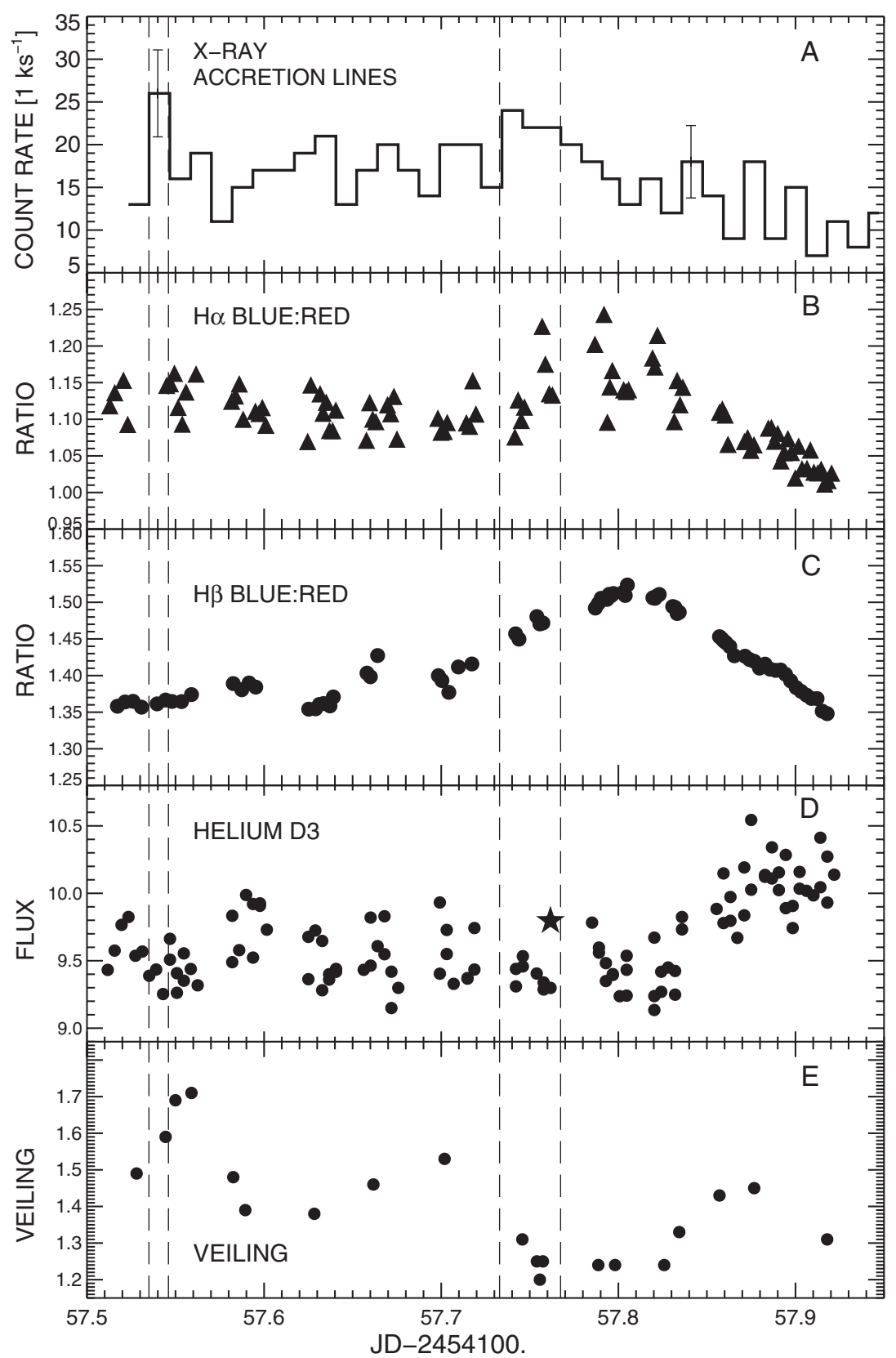

Figure 12. Changes in the profile characteristics of the $\mathrm{H} \alpha$ and $\mathrm{He}$ I (D3, 5876 $\mathrm{A})$ lines during an "accretion event" as indicated by the increase in flux of X-ray line emission (binned at $1 \mathrm{ks}$ ) formed in the accretion shock (panel A). Panel B: the total flux of H $\alpha$ normalized to the continuum does not change (see Figure 11), but the flux ratio of a $1 \AA$ band on the short (blue) and long (red) wavelengths of the $\mathrm{H} \alpha$ emission displays an increased asymmetry near day 57.75. Panel C: flux ratio of the $\mathrm{H} \beta$ line, short wavelength to long wavelength measured in similar fashion as $\mathrm{H} \alpha$. Panel D: the continuum-normalized flux of the He I D3 line ( $\lambda 5876)$. The star symbol marks the spectrum taken at 06:18 (Day 57.763) where the wings of the helium line have broadened (see Figure 14). Veiling (panel E) is also shown (described in Section 9).

et al. (2004). This interpretation relies on the assumption that the entire $\mathrm{H} \alpha$ line arises from accreting material, and it is obvious from our observations that the wind plays a significant role in modifying these profiles. The largest variation occurs on the short-wavelength side of the profile, at negative velocities, and is not consistent with motions expected from an accretion stream. Thus the values of accretion inferred from the widths may not be meaningful.

\section{ACCRETION EVENTS}

During the Chandra pointing, three "accretion events," signaled by an abrupt short-lived increase in the flux of the X-ray accretion lines occurred at day 48.2, day 57.54 (in 1 ks binning), and day 57.74. These are marked by arrows in Figure 5. Details of these events and the spectroscopic response are shown in Figure 11 (with the X-rays binned at $3 \mathrm{ks}$ ) and Figure 12 (X-rays binned at $1 \mathrm{ks}$ ).

WASP photometry did not coincide with the first accretion event and only one contemporaneous spectrum is available. The second accretion event is characterized by a sharp short (1 ks) rise in the accretion-line flux by a factor of two. The third accretion event occurred $\sim$ day 57.74 as marked by an abrupt increase $(\sim 26 \%)$ in the flux of the X-ray accretion lines that lasts for $3 \mathrm{ks}$. This event marks the highest hourly flux of accretion 

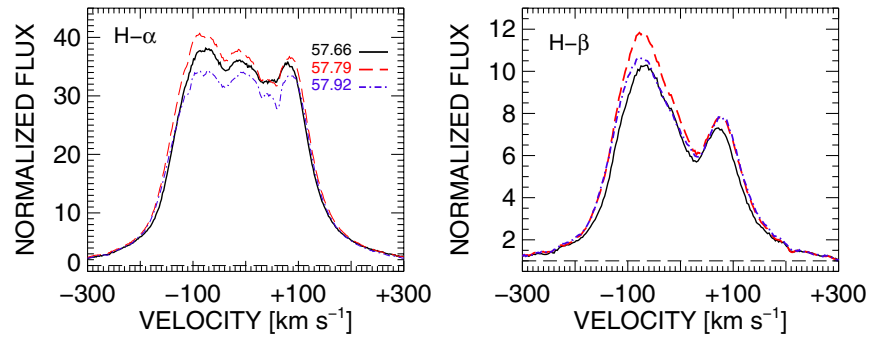

Figure 13. Profiles from Magellan spectra that sample the time (Day 57.66) before the long X-ray accretion event, the onset of $\mathrm{H} \alpha$ asymmetry (Day 57.79) noted in Figure 12, and at the end of the observing night (Day 57.92). Left panel: $\mathrm{H} \alpha$-the total line flux increases slightly between 57.66 and 57.79 in these spectra, but Figure 11 shows this increase is not significant. At the end of the observing night (marked 57.92), the $\mathrm{H} \alpha$ line flux is less and the profile more symmetric. Right panel: $\mathrm{H} \beta$ profile showing a similar flux change and the signature of constant inflow. Both emission lines show increased blue asymmetry indicating increased downflow directly after the X-ray accretion event, but this blue asymmetry decreases starting at day 57.8 , about $1.5 \mathrm{hr}$ after the X-ray accretion event.

(A color version of this figure is available in the online journal.)

lines that occurred during the extent of the long $500 \mathrm{ks}$ Chandra observation.

We first examine the long event on day 57. Fortuitously, it occurred during intensive spectral monitoring. As shown in Figure 11, the total $\mathrm{H} \alpha$ flux did not change, but enhancement occurs in the blue and red wings of $\mathrm{H} \alpha$ as well as a blue asymmetry in the line profile. Additionally the $\mathrm{H} \beta$ flux increased, with the blue side of the $\mathrm{H} \beta$ line showing greater enhancement (Figure 11, panels D and E).

A detailed examination of the accretion profiles reveals the time sequence of this event. Inspection of the $1 \mathrm{ks}$ binning of accretion lines (Figure 12) shows that the abrupt increase starts at 05:46 UT (Day 57.74), where we take the midpoint of the $1 \mathrm{ks}$ bin. The $\mathrm{H} \alpha$ asymmetry changes after 05:55 UT, nine minutes later. The $\mathrm{H} \beta$ line also exhibits an increasing blue asymmetry peaking slightly after the $\mathrm{H} \alpha$ line. The sequential and abrupt profile changes following $X$-ray accretion suggests $H \alpha$ and $H \beta$ originate in the downflowing post-shock cooling region. These changes provide strong evidence too that the increase of the X-ray accretion component represents an actual increased accretion rate and not a decrease in the column density of accreting material. The latter process could reduce the soft $\mathrm{X}$-ray absorption and thereby strengthen the observed flux of the accretion lines. The veiling (discussed in Section 9) also increases following this event, but is delayed by $\sim 2 \mathrm{hr}$.

$\mathrm{H} \alpha$ profiles taken before (Day 57.66) during (Day 57.79) and at the end of the event (Day 57.92) are shown in Figure 13. All parts of the profile behave similarly, suggesting that the emission measure of a turbulent region increased, and considerable variability occurs in the total $\mathrm{H} \alpha$ flux as well.

The helium line profile (Figure 14) changes quite dramatically beginning at the same time as the $\mathrm{H} \alpha$ asymmetry change. The helium line is known to have a broad and narrow component and the broad component is frequently identified as an accretion signature (Muzerolle et al. 1998b; Alencar \& Batalha 2002; Donati et al. 2011). Before a significant increase in the total line flux, the profile begins to broaden, particularly on the positive velocity wing (between +100 and $+200 \mathrm{~km} \mathrm{~s}^{-1}$ ). The He I ( $\lambda$ 5876) line has not changed in flux at 06:16 UT (Day 57.762); however, the narrow-line component increases and the wing broadening appears in the spectrum at 06:18 (Day 57.763, about 30 minutes after the increase in the X-ray accretion

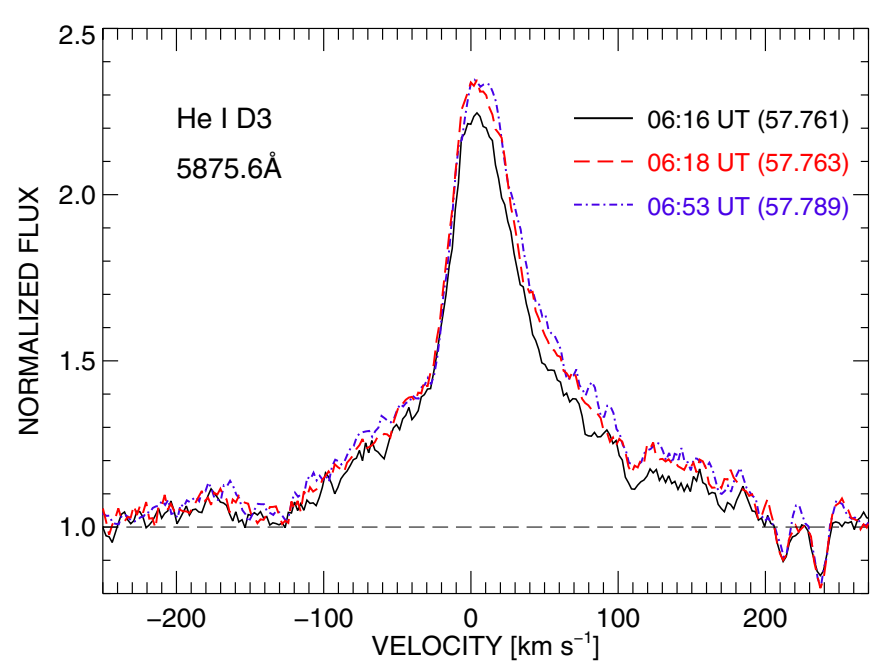

Figure 14. He I D3 line directly following the change in the $\mathrm{H} \alpha$ line asymmetry. The local UT time (2007 February 26) of mid-exposure is noted for the three spectra, and the mid-exposure time of the spectrum obtained at 06:18 UT (Day 57.763 ) is marked by a star in Figure 12.

(A color version of this figure is available in the online journal.)

lines). The flux of the He I $5876 \AA$ line (Figure 12) increases about $2.5 \mathrm{hr}$ after the X-ray accretion event. Decomposing the profiles into a narrow and broad component, we find, at the end of the observing night compared to the profile earlier in the night, that the narrow component remains at constant flux (to within 15\%) and the FWHM to within $1 \mathrm{~km} \mathrm{~s}^{-1}$, while the broad component increased both in flux by $30 \%$ and width by $7 \mathrm{~km} \mathrm{~s}^{-1}$, and its redshift increased from +15 to $+21 \mathrm{~km} \mathrm{~s}^{-1}$ with respect to the narrow component. Interpretation of this redshift must be treated with caution because increased shortwavelength absorption can result from outflowing material (cf. Section 8) and cause an apparent redshift in line position. The broad (Gaussian) component has an FWHM of $175 \mathrm{~km} \mathrm{~s}^{-1}$ on average. This width exceeds the thermal width of helium at $10^{4} \mathrm{~K}\left(11 \mathrm{~km} \mathrm{~s}^{-1}\right)$, and is similar to the wide lines observed in $\mathrm{Ne} \mathrm{X}$ and $\mathrm{H} \alpha$. Therefore we also associate the broad component of He I with the turbulent post-shock region.

The earlier short accretion event (Day 57.54) shows X-ray enhancement for a $1 \mathrm{ks}$ interval. The $\mathrm{H} \beta$ flux and the red and blue segments of $\mathrm{H} \alpha$ are decreasing at the start of the optical observations, but we have no knowledge of prior activity in the X-ray emission. The ASAS photometry (Figure 4) hints that flaring may have occurred near day 57.66. Subsequently, and quickly, the veiling increases sharply, followed by an increase in the flux of the helium line. This event exhibits a substantially smaller impact on the spectroscopic diagnostics than the later accretion event discussed above, perhaps because of its shorter extent in time.

\section{CORONAL FLARE EVENT}

A flare occurred at day 58.2 (Figure 15). The $\mathrm{H} \alpha$ profile was measured once during the flare at the SAAO, and the line flux was comparable to earlier values. Spectra over the next $5 \mathrm{hr}$ exhibited a decrease in the $\mathrm{H} \alpha$ equivalent width by $\sim 8 \%$, attributed to the decrease in strength of the short-wavelength side of the profile, similar to that shown in Figure 8 between days 58.5 and 58.92. The WASP optical photometry suggests flaring events occurred during day 55, and the SAAO photometry 


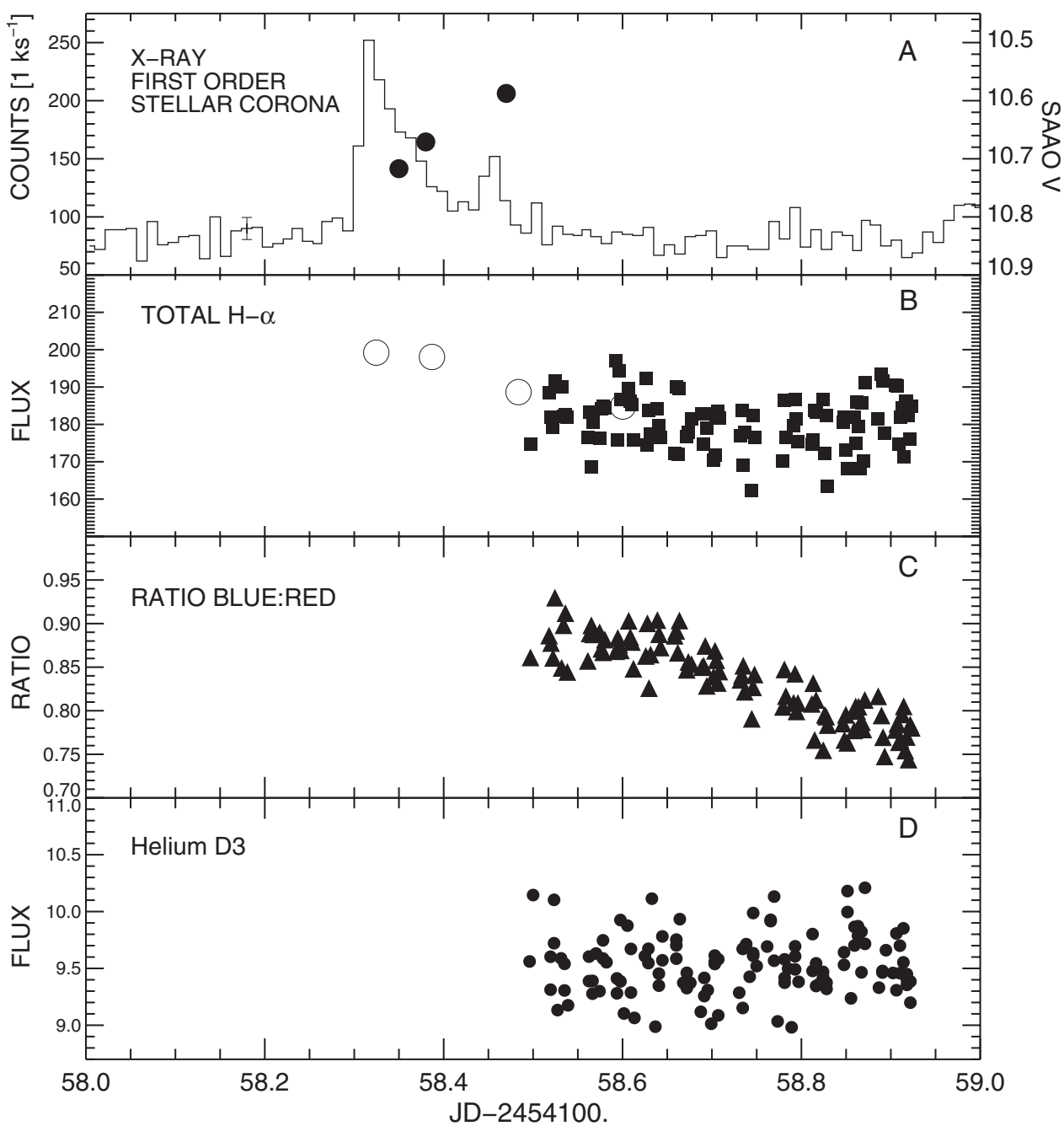

Figure 15. $\mathrm{H} \alpha$ characteristics near the coronal flaring event. Panel A: total X-ray flux (corona) binned in $1 \mathrm{ks}$ intervals at the time of the coronal flare. The filled circles represent the simultaneous SAAO photometry with the scale on the right-hand side axis. Panel B: the total flux in the H $\alpha$ line as measured in the spectra from the SAAO $(\bigcirc)$ and spectra taken at Magellan ( $\square$ ). Panel C: ratio of the strength of the blue wing to the red wing of the H $\alpha$ line measured from the Magellan spectra. Panel D: flux of the He I emission line at $\lambda 5876$. These line characteristics differ from those found following the accretion event shown in Figure 12; a distinct accretion event is not seen in the X-ray data at this time (see Figure 7).

indicates that TW Hya was brighter then too. However, the SAAO $\mathrm{H} \alpha$ equivalent width remains low during that event.

We conclude that the flare affected the V-band photometry of TW Hya, causing an optical brightening (a "white-light flare"), but does not change the flux nor the characteristics of the $\mathrm{H \alpha}$ profiles. This flare appears to be a stellar coronal event unrelated to the accretion process.

\section{THE WIND IN HELIUM $\lambda 10830$}

The near-IR He I $10830 \AA$ line is formed in the stellar atmosphere and wind, and its metastable nature allows a good probe of stellar winds especially in T Tauri stars (Edwards et al. 2003; Dupree et al. 2005). One observation in 2002 (Dupree et al. 2005) showed the wind extending to a speed of $-280 \mathrm{~km} \mathrm{~s}^{-1}$; in 2005 the terminal velocity reached $\sim 300 \mathrm{~km} \mathrm{~s}^{-1}$. A spectrum obtained on 2007 March 1 (Figure 16) with PHOENIX at Gemini-S shows that wind opacity is larger than previously observed, particularly at velocities greater than $-200 \mathrm{~km} \mathrm{~s}^{-1}$ and the terminal velocity is slightly higher $\left(\sim 325 \mathrm{~km} \mathrm{~s}^{-1}\right)$. The emission component of $\lambda 10830$ is substantially weaker as well. In addition, subcontinuum absorption at positive velocities is stronger then measured previously and extends to $+300 \mathrm{~km} \mathrm{~s}^{-1}$.
An optical spectrum taken at Magellan 60 minutes prior to the PHOENIX observation is instructive (Figure 17). Not only are the $\mathrm{H} \alpha$ and $\mathrm{H} \beta$ lines weakened from the previous three nights (cf. also Figure 8), but the profiles are characterized by wind absorption. In fact, the $\mathrm{He}$ I $\lambda 5876$ transition and $\mathrm{H} \beta$ both exhibit a narrow wind absorption feature at $\sim-125 \mathrm{~km} \mathrm{~s}^{-1}$. The $\lambda 5876$ transition arises from the upper ${ }^{3} \mathrm{P}$ level of the $\lambda 10830$ near-IR line, and absorption by the wind is similar to that found in the $\lambda 10830$ profile, although not extending to such high velocities. Higher speeds observed in the near-IR He I line than $\mathrm{H} \beta$ suggest an accelerating outflow. Clearly, both the wind speed and opacity vary with time, but insufficient observations prevent a direct association of the near-IR He I line profile with the accretion process.

\section{VEILING}

Veiling, which describes the addition of a continuum spectrum to the stellar spectrum, is thought to occur in TW Hya due to the presence of an accretion "hot spot" (Alencar \& Batalha 2002). Magellan spectra taken during the nights of 2007 February 26 and 27 (Days 57 and 58) were used to determine the veiling because they were simultaneous with the Chandra 


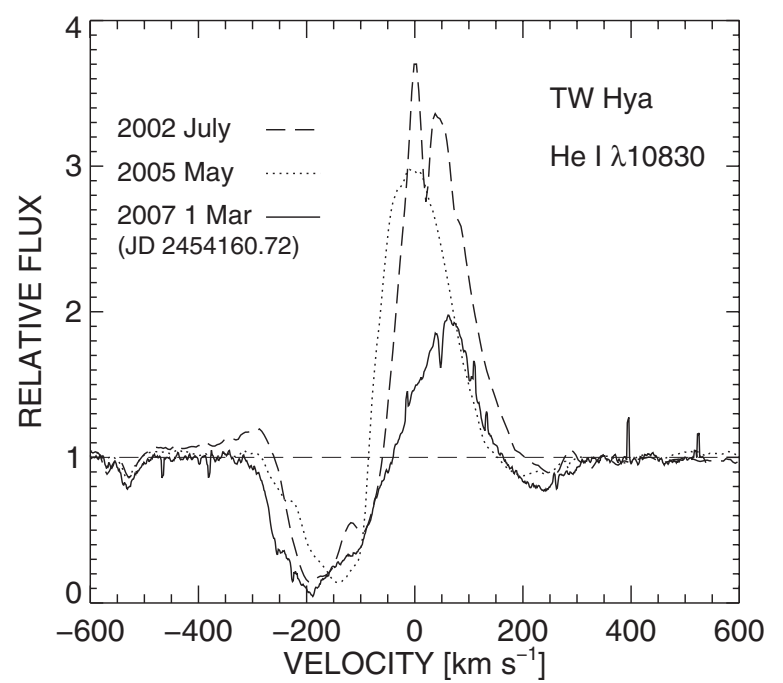

Figure 16. PHOENIX spectrum of He I, $10830 \AA$ obtained at Gemini-S on 2007 March 1 (JD 2454160.5), as compared to spectra obtained previously with Keck/NIRSPEC at slightly lower resolution (Dupree et al. 2005). The emission in 2007 is substantially weaker, and wind absorption extends to higher velocities than in previous observations. Stronger absorption occurs below the continuum on the long-wavelength side of the line. The total extent of the line appears to be $\pm 325 \mathrm{~km} \mathrm{~s}^{-1}$.

pointing. The $\mathrm{T}$ Tauri star spectrum is modeled as a normal stellar photosphere plus a smoothly varying veiling continuum. The normal photosphere is taken from a spectrum of GJ1172, a K7V star obtained at Magellan (1200 s exposure) during the February campaign. This template star was used previously by Alencar \& Batalha (2002) who state that it "presented a perfect match to the photospheric lines of TW Hya, after it was rotationally broadened to the TW Hya $v \sin i$ and artificially veiled." The MIKE spectra of GJ1172 when rotationally broadened with $v \sin i=5 \mathrm{~km} \mathrm{~s}^{-1}$, confirm that it is a good match for TW Hya.

We follow the procedures outlined by Hartigan et al. (1989) by constructing a rotationally broadened normalized spectrum of GJ 1172 to use as a template. Many spectral regions, ranging in width from 5 to $10 \AA$ were selected from the centers of echelle orders on the blue and red side of the MIKE spectra. The observed normalized TW Hya spectrum, $\mathrm{O}(\lambda) / \mathrm{O}(\lambda)_{\text {cont }}$, is related to the normalized template spectrum, $T(\lambda) / T(\lambda)_{\text {cont }}$ :

$$
\frac{O(\lambda)}{O(\lambda)_{\mathrm{cont}}}=\frac{\left[\frac{T(\lambda)}{T(\lambda)_{\mathrm{cont}}}+r\right]}{[1+r]},
$$

where $r$ represents the fractional veiling that is produced by the veiling continuum, $V$ :

$$
r=\frac{V(\lambda)_{\mathrm{cont}}}{T(\lambda)_{\mathrm{cont}}}
$$

By changing the value of $r$, the right-hand side of Equation (1) becomes an artificially veiled template spectrum to be compared to the observed TW Hya spectrum yielding the veiling in the selected regions. Iterations through $r$ values in steps of 0.1 allow the best fit to be determined by seeking a minimum value of $\chi^{2}$ between the template and the TW Hya spectrum. Values of the veiling $(r)$ as a function of wavelength are shown in Figure 18 for five spectra spanning $5 \mathrm{hr}$. The increased veiling toward shorter wavelengths is well known (Alencar \& Batalha 2002) and expected from a continuum source with $T \approx 8000 \mathrm{~K}$.

During this time, the veiling varies, which appears to result principally from the changing size of the accretion hot spot.

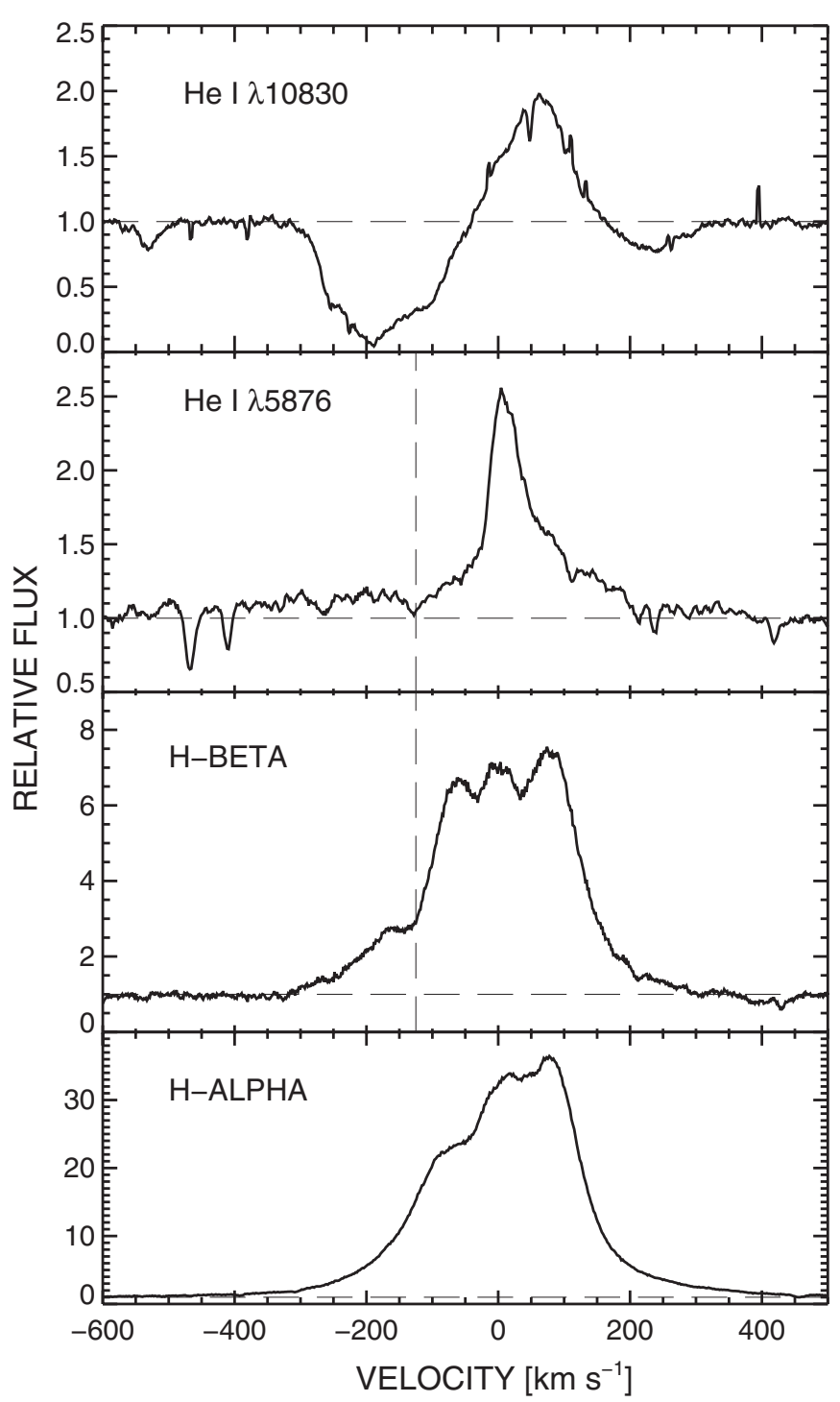

Figure 17. Three major diagnostic emission lines observed within an hour of the He I near-infrared $10830 \AA$ transition on day 60 . Evidence of an outflowing wind is apparent in all lines with outflow velocities increasing from $\mathrm{H} \beta$ and the $5876 \AA$ line to the $10830 \AA$ transition. Inflow can also be noted in the subcontinuum absorption of the $10830 \AA$ line.

In order to search for a relation between the veiling values and the X-ray flux, we introduce an average value of the veiling based on seven measurements between $4400 \AA$ and $5000 \AA$ taken from the same regions in all spectra. These are the values used previously in Figure 12; the complete set is given in Table 3 and shown in Figure 19 (upper panels) aligned with the simultaneous measures of the total X-ray flux. The veiling values and $\mathrm{X}$-ray fluxes were iterated through positive time shifts from 0 to 0.5 days in increments of 0.01 days. The correlation between the veiling and the total flux is strongest when the veiling measures are shifted by +0.11 days (lower panels) as shown in Figure 19, giving a correlation coefficient of 0.78 . The correlation between the veiling parameter and the simultaneously measured total $\mathrm{X}$-ray flux is shown in Figure 20.

The principal source of the total X-ray flux is most likely the corona. Our models based on the Chandra spectrum (Brickhouse et al. 2010) indicate that most of the total X-ray flux arises from the corona. In order to confirm this conclusion, we selected only the highest energy component of the total flux, 


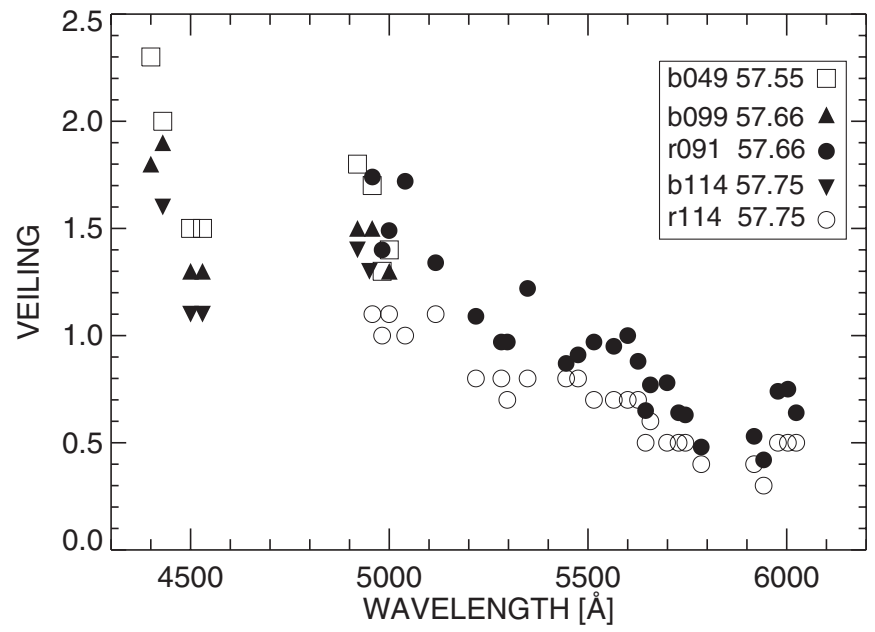

Figure 18. Veiling as a function of wavelength during the night of 2007 February 26 (UT). The spectrum (either blue (b) or red (r) side of MIKE, and the day (JD-245100) are noted). The veiling increases toward shorter wavelengths and the value of the veiling decreases during the first part of the night. The "gap" between $4600 \AA$ and $4800 \AA$ occurs because photospheric lines in this region are shallow, and it is difficult to determine a value of the veiling.

Table 3

Blue Veiling Parameter

\begin{tabular}{lccccc}
\hline \hline $\begin{array}{l}\text { Day } \\
(J D-2454100)\end{array}$ & Veiling & Spectrum & $\begin{array}{c}\text { Day } \\
\text { (JD-2454100) }\end{array}$ & Veiling & Spectrum \\
\hline 57.5280 & 1.49 & b044 & 57.8259 & 1.24 & b138 \\
57.5444 & 1.59 & b047 & 57.8344 & 1.33 & b143 \\
57.5500 & 1.69 & b049 & 57.8571 & 1.43 & b148 \\
57.5590 & 1.71 & b050 & 57.8767 & 1.45 & b157 \\
57.5826 & 1.48 & b069 & 57.9179 & 1.31 & b170 \\
57.5894 & 1.39 & b071 & 58.5207 & 1.23 & b050 \\
57.6284 & 1.38 & b090 & 58.5995 & 1.24 & b068 \\
57.6617 & 1.46 & b099 & 58.6650 & 1.35 & b091 \\
57.7019 & 1.53 & b106 & 58.6974 & 1.31 & b102 \\
57.7458 & 1.31 & b114 & 58.7388 & 1.26 & b114 \\
57.7540 & 1.25 & b115 & 58.7848 & 1.30 & b131 \\
57.7557 & 1.20 & b116 & 58.8181 & 1.23 & b143 \\
57.7574 & 1.25 & b117 & 58.8534 & 1.54 & b166 \\
57.7887 & 1.24 & b121 & 58.8969 & 1.73 & b181 \\
57.7982 & 1.24 & b127 & & & \\
\hline
\end{tabular}

namely, that greater than $1 \mathrm{keV}$, to correlate with the veiling. The same delayed shift of 0.11 days gives the highest correlation coefficient, with a value of 0.72 , only slightly lower than the correlation with the total flux. This suggests that the corona responds to the increase in veiling produced $\sim 2.5 \mathrm{hr}$ earlier. We searched for similar correlations between line components of the X-ray spectrum itself without definitive results. Taking the line spectrum divided into accretion and coronal components does not yield a significant correlation with appropriate lag between the components themselves and the veiling. For this, we evaluated small negative as well as positive lags between the accretion-line flux and the veiling values. Because the counts are low in the accretion and coronal line components and affected by the underlying continuum, any correlation would be weakened.

\section{A SUGGESTED MODEL}

These results suggest that the accretion process can be traced spectroscopically in the following way. Material channeled along the magnetic flux tubes threaded from the circumstel- lar disk accelerates to supersonic velocities and creates a shock near the stellar surface (Figure 21). Brickhouse et al. (2010) identified emission features in the Chandra X-ray spectrum that originate in the MK shocked plasma. The shock size as estimated from the emission measure and electron densities derived from the X-ray spectrum has a scale length of $3.2 \times 10^{4} \mathrm{~km}$. The shocked material cools in a turbulent plasma as it approaches the stellar photosphere and the profiles of the optical emission lines mark the progress of the event. Following the major accretion enhancement (Day 57.74) noted in Figures 11 and 12, the $\mathrm{H} \alpha$ and $\mathrm{H} \beta$ line profiles show a signature of abruptly increasing downflow (toward the star) revealed by the increase in blue:red asymmetry. It is important to note that both Balmer series lines are broad, and the line wings are roughly symmetric. The FWHM of these lines reaches $\sim \pm 150 \mathrm{~km} \mathrm{~s}^{-1}$, which indicates substantial turbulent broadening in excess of the thermal width $\left(\sim 21 \mathrm{~km} \mathrm{~s}^{-1}\right)$, and is comparable to the turbulent velocity inferred from the breadth of the Ne IX lines in the post-shock accretion zone, namely, $165 \mathrm{~km} \mathrm{~s}^{-1}$ measured in the Chandra spectrum (Brickhouse et al. 2010). Additionally, hydrogen emission lines in the infrared (Vacca \& Sandell 2011) display broadened line wings amounting to several hundred $\mathrm{km} \mathrm{s}^{-1}$, consistent with the $\mathrm{H} \alpha$ profiles.

The He I $\lambda 5876$ transition exhibits an increase in the broad wing component after the downflow signatures occur in the Balmer lines. This broadening occurs over a timescale of minutes (Figure 14). The veiling and the He I flux increase about 0.08 days $(\sim 2 \mathrm{hr}$ ) after the accretion event marked by $\mathrm{X}$-rays. Such a timescale is roughly consistent with the timescale suggested by the inflow velocity and size of the hot spot. The size of the photospheric "hot spot" on TW Hya has been estimated to cover $2 \%-6 \%$ of the apparent stellar disk, although at times it extends from the pole to lower latitudes and the area coverage increases to 25\% (Costa et al. 2000; Batalha et al. 2002; Donati et al. 2011). Simulations of disk accretion (Romanova et al. 2004) suggest coverage values ranging from $1 \%$ to $20 \%$. We take $10 \%$ for this example, and for a stellar radius of $0.8 R_{\odot}$, the hot spot has a radius of $3.5 \times 10^{5} \mathrm{~km}$. For a velocity of $35 \mathrm{~km} \mathrm{~s}^{-1}$ corresponding to the $\mathrm{H} \beta$ absorption feature, this suggests a characteristic timescale of $2.8 \mathrm{hr}$, which is in harmony with the observed change in the veiling. Following the changes in photospheric veiling, the coronal X-rays respond to this increase with a delay on average of 0.11 days ( $2.6 \mathrm{hr}$, Figure 20). These results give strong evidence for an accretion-fed corona which may contribute to the wind acceleration as well.

It is tempting to attribute the absence of a wind followed by the increase in wind opacity as a consequence of the accretion event. One could envision a dramatic change in the magnetic field configuration caused by a sharp increase in the amount of accreting material, allowing a wind to develop. However, the rotation of the star over several days could also present a different aspect of an asymmetric accreting geometry and outflow.

\section{COMPARISON WITH PREVIOUS RESULTS}

Our model for TW Hya suggests that the contribution to $\mathrm{H} \alpha$ from the post-shock cooling region must overwhelm any contribution from the accretion stream. Previous models have not considered the contribution from the chromospheric or postshock material. Muzerolle et al. (2000) assume that all of the $\mathrm{H} \alpha$ emission arises in the hot stream of material extending out to a few $R_{\star}$, channeled from the circumstellar disk by the magnetic field, and free-falling to produce the accretion shock. Another 

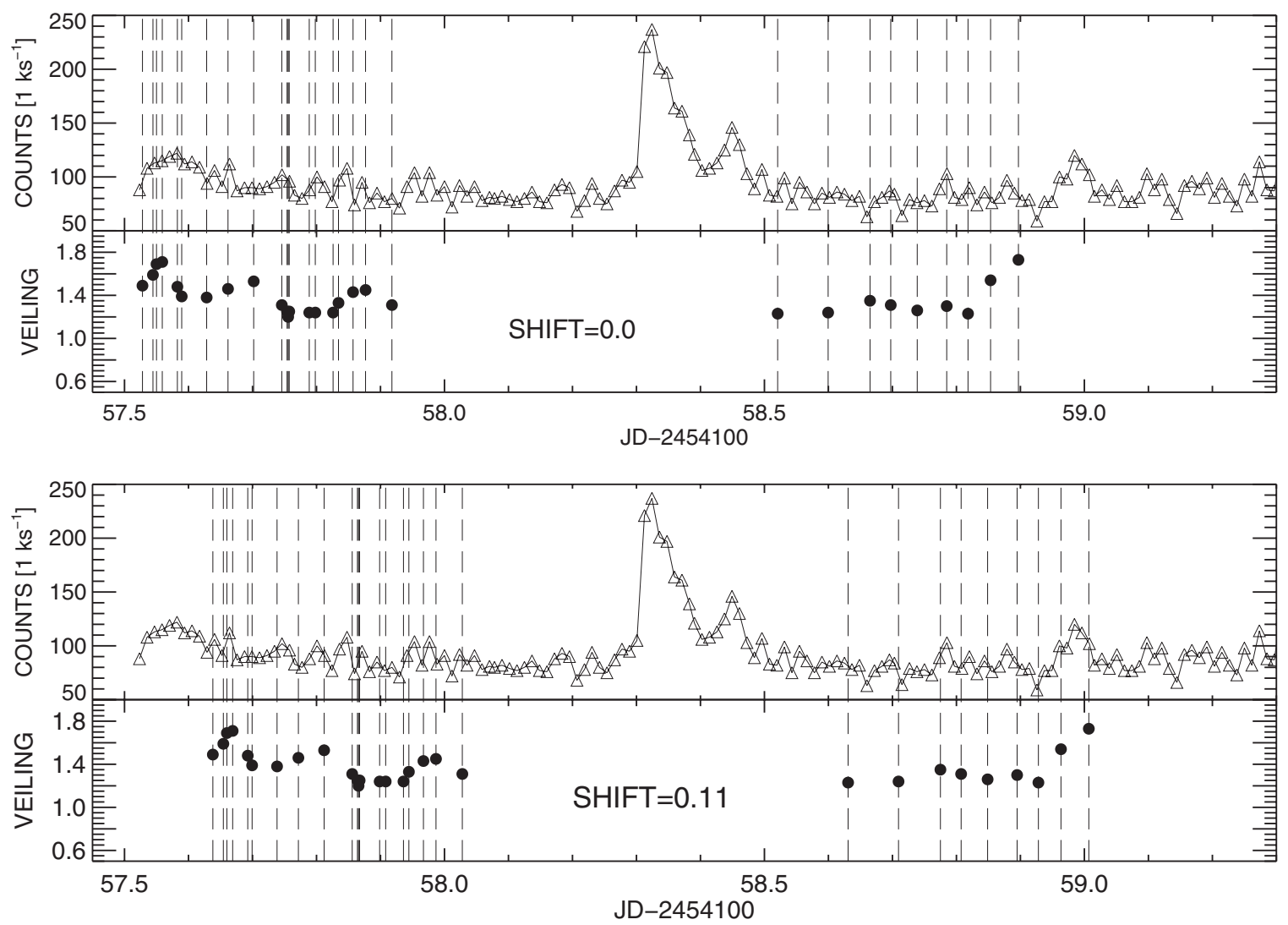

Figure 19. Total (coronal) X-ray flux and average blue veiling with no time shift (top two panels) and a time shift of 0.11 days applied to the veiling measures (bottom two panels), suggesting that the appearance of a hot photospheric region (indicated by veiling) precedes the increase in coronal X-ray flux. Error bars ( $1 \sigma$ ) for the $\mathrm{X}$-ray flux measures are smaller than the symbol size.

paper (Muzerolle et al. 2001) explicitly shows in more detail the geometry that is generally assumed. These papers do not include a contribution to the $\mathrm{H} \alpha$ profile from the post-shock cooling zone or the chromosphere. A simple calculation demonstrates that the post-shock/chromospheric region has substantially more material than the plasma in the accretion stream, and most likely will dominate formation of the $\mathrm{H} \alpha$ profile.

Mass conservation (Brickhouse et al. 2010) yields the preshock electron number density $\left(5.7 \times 10^{11} \mathrm{~cm}^{-3}\right)$, and the filling factor of the shock (1.1\% of the stellar surface) based on a dipole magnetic field (Cranmer 2008). Assuming a truncation radius for the gas disk of $4.5 R_{\star}$ where $R_{\star}=0.8 R_{\odot}$, we estimate an upper limit to the emission measure of a (cylindrical) channeling tube of length $3.5 R_{\star}$ as $N_{e}^{2} \times V=2.8 \times 10^{55} \mathrm{~cm}^{-3}$, where $V$ is the emitting volume. This is an upper limit for several reasons. The density falls off toward the disk by a factor of 10 in the Muzerolle et al. (2001) models, and the accretion streams have a length of only $1.2-2 R_{\star}$, decreasing the emission measure to $\sim 2 \times 10^{54} \mathrm{~cm}^{-3}$ or less.

For comparison, what is the expected chromospheric emission measure resulting from the accretion shock? Modeling gives the post-shock electron density as $2.5 \times 10^{15} \mathrm{~cm}^{-3}$ at the bottom of the chromosphere where the accretion stream ram pressure matches the gas pressure. We take the density of $7.7 \times 10^{13} \mathrm{~cm}^{-3}$, which is the mean between the immediate postshock density and the value at the base of the chromosphere, and a length scale of half the post-shock cooling distance, namely, $200 \mathrm{~km}$ (Brickhouse et al. 2010). The area of the chromospheric/ photospheric hot spot appears to be $\sim 3 \%$ (Donati et al. 2011) of the stellar surface $\left(1.2 \times 10^{21} \mathrm{~cm}^{2}\right.$ for a star with $\left.R=0.8 R_{\odot}\right)$.
The emission measure $N_{e}^{2} \times V \sim 1.4 \times 10^{56} \mathrm{~cm}^{-3}$ is a factor of $\sim 100$ larger than the emission measure from the accretion stream. Another estimate of the emission measure of the hot region at the accretion footpoints responsible for the near-infrared hydrogen Paschen series emission in TW Hya (Vacca \& Sandell 2011) gives an emission measure of $0.7-4 \times 10^{55} \mathrm{~cm}^{-3}$, a value larger than from the accretion stream and compatible with the characteristics derived above for the post-shock cooling region.

What might be the effects of a deviation from a dipole magnetic configuration? Donati et al. (2011) suggest that the star may have an octupolar component to the magnetic field in the photosphere, but an octupole field falls off exceedingly rapidly $\left(r^{-5}\right)$ with distance from the star compared to a dipole $\left(r^{-3}\right)$ and is generally thought not to extend far enough to penetrate the circumstellar disk. In any case, the octupole field suggested by Donati et al. has a pole (roughly) coincident with the stellar pole where accretion is believed to occur. Thus if accretion toward the pole proceeded on a octupole field line, it would be hard to differentiate from a dipole configuration. However, in our view, the accretion stream is not the source of the $\mathrm{H} \alpha$ profile, as discussed previously.

The observed turbulent motions are compatible with cooling zone parameters derived from the X-ray observations. Densities in the cooling zone range from $2 \times 10^{15}$ to $2.3 \times 10^{12} \mathrm{~cm}^{-3}$ and temperatures from several $\mathrm{MK}$ in the immediate post-shock region. down to $\sim 10^{5} \mathrm{~K}$. Donati et al. (2011) suggest that the octupolar component of the photospheric field at the pole reaches a maximum of $2.5-2.8 \mathrm{kG}$, whereas the dipole field varies from 0.4 to $0.7 \mathrm{kG}$. In the photosphere, the magnetic pressure caused by the octupole field would dominate the gas 

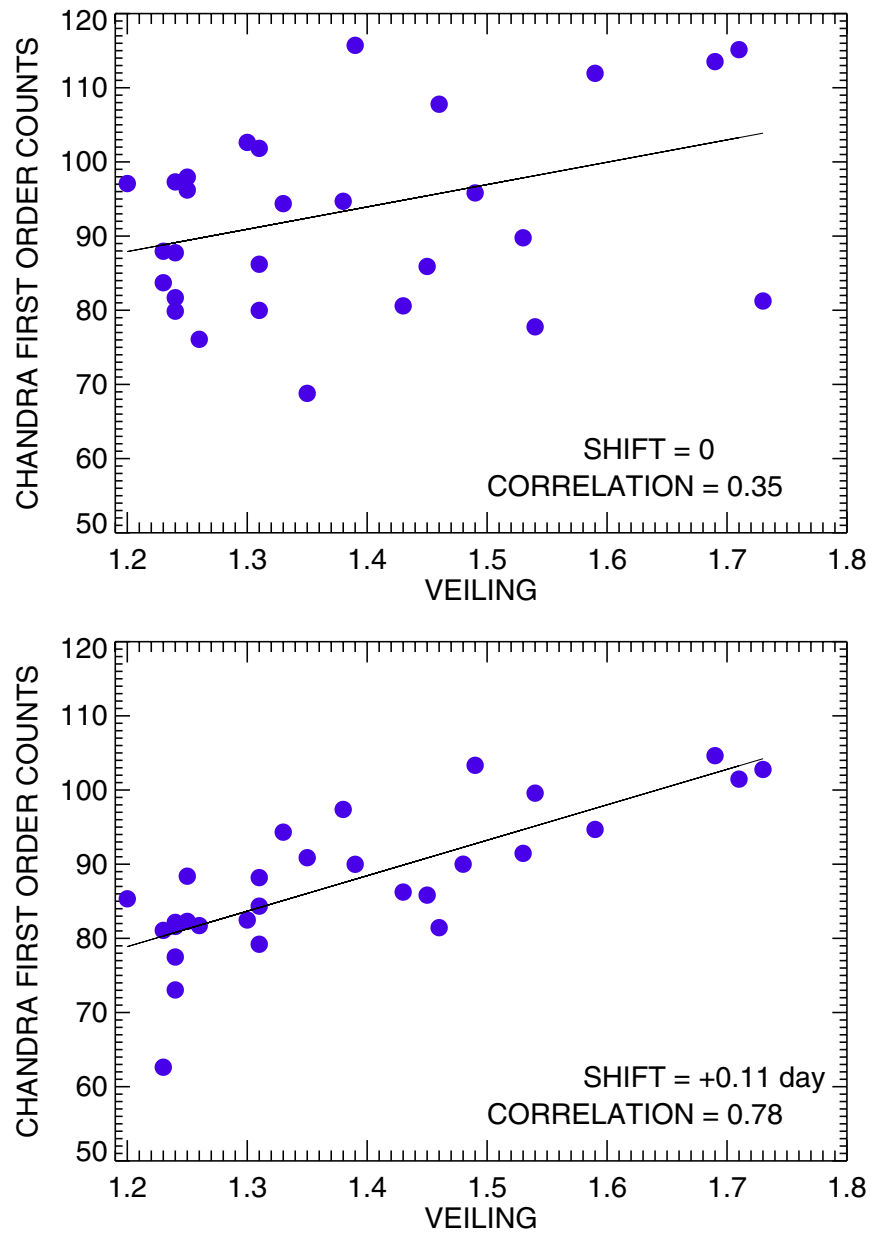

Figure 20. Total first-order Chandra counts interpolated to the times of the spectra as a function of the average blue veiling (4500-5000 $\AA^{2}$. Top panel: no time shift between veiling measure and coronal X-ray flux. Lower panel: the veiling measure is shifted by +0.11 days where the agreement between the coronal flux and veiling is substantially improved. This suggests that the corona responds 0.11 days $(\sim 2.6 \mathrm{hr})$ later to an increase in the veiling.

(A color version of this figure is available in the online journal.)

pressure at an effective temperature of $4000 \mathrm{~K}$. An octupole field falls off rapidly $\left(r^{-5}\right)$ and this octupole field strength becomes commensurate with the dipole field at $2 R_{\star}$. For heights greater than $0.2 R_{\star}$ above the photosphere, at a temperature of $10,000 \mathrm{~K}$ in a dense post-shock region, the gas pressure becomes comparable to the magnetic pressure from an octupole field. Other measures of the magnetic field (Yang et al. 2007) suggest that the field is substantially smaller than indicated by the Donati et al. (2011) model, and pressure balance would occur even closer to the photosphere. However, if MHD turbulence is present, the strong fields do not impede large motions in the plasma, and the observed turbulence could be present at any level above the photosphere.

We note that accretion shock modeling to date has been unable to reproduce both the profiles and the intensities of the observed ultraviolet lines from TW Hya (Günther \& Schmitt 2008; Lamzin et al. 2007). The emission measures of the postshock regions discussed here are large, much larger than found in a normal $\mathrm{K} 7 \mathrm{star}$, and would be expected to form a strong $\mathrm{H} \alpha$ line. We can estimate the expected $\mathrm{H} \alpha$ flux based on the observed Paschen series lines which terminate on the $n=3$ level of hydrogen, the upper level of the Balmer $\mathrm{H} \alpha$ line. A sequence of lines has been measured in TW Hya (Vacca \& Sandell 2011),

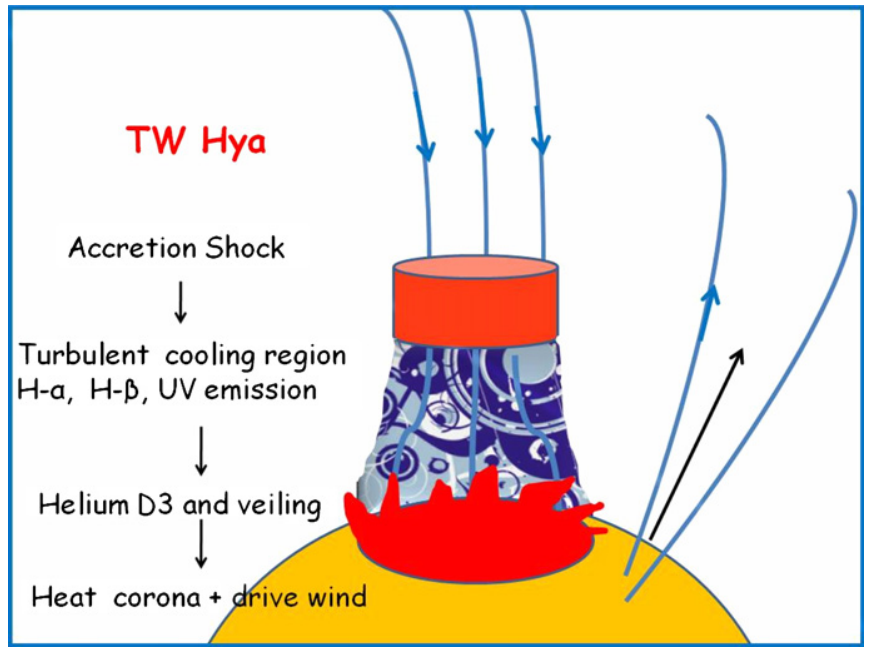

Figure 21. Schematic cartoon suggesting the sequential steps in the accretion process.

(A color version of this figure is available in the online journal.)

but this does not include the strongest line in the series, the Paschen- $\alpha$ transition. Assuming that the total number of photons landing in the $n=3$ level produces the $\mathrm{H} \alpha$ line, we find an $\mathrm{H} \alpha$ flux of $7.7 \times 10^{-12} \mathrm{erg} \mathrm{cm}^{-2} \mathrm{~s}^{-1}$ which represents a lower limit because the contribution of the Paschen- $\alpha$ line is not included. Rucinski \& Krautter (1983) estimate the $\mathrm{H} \alpha$ flux from TW Hya to be: $1.6 \times 10^{-11} \mathrm{erg} \mathrm{cm}^{-2} \mathrm{~s}^{-1}$, which is in harmony with the value from the infrared emission. Thus the post-shock material inferred from the Paschen series (Vacca \& Sandell 2011) can produce the observed $\mathrm{H} \alpha$ flux. The size of this region depends on the density. For a temperature $\gtrsim 7500 \mathrm{~K}$, a density of $10^{12} \mathrm{~cm}^{-3}$, and a $4 \%$ covering fraction, Vacca \& Sandell (2011) find that the thickness ranges between $10^{4}$ and $10^{5} \mathrm{~km}$; this value decreases to $340-820 \mathrm{~km}$ if the density is an order of magnitude larger. Although current theoretical models of post-shock conditions generally focus on temperatures producing X-ray emission, a one-dimensional post-shock model (Sacco et al. 2010) contains chromospheric regions at temperatures of $8000 \mathrm{~K}$ with a thickness of $\sim 1500 \mathrm{~km}$, and densities of $10^{15} \mathrm{~cm}^{-3}$. This is denser and may be thinner than indicated by the Paschen series measures. Additionally, the models remain static at these chromospheric temperatures in contrast to the motions inferred from the changing profile shapes observed in $\mathrm{H} \alpha$.

The observations reported here suggest that the broad (symmetric) $\mathrm{H} \alpha$ line arises from a large turbulent region that appears to be part of the post-shock cooling region. Such an $\mathrm{H} \alpha$ volume is not considered in current model calculations and these observations can empirically define its characteristics. It may be associated with the large $\mathrm{O}$ VII region that is extrinsic to the currently postulated post-shock region.

\section{CONCLUSIONS}

This comprehensive study clarifies the accretion process, and demonstrates the power of simultaneous spectral diagnostics and photometry to probe the plasma dynamics in TW Hya. We find:

1. The periodicity in the optical photometry appears unrelated to the $\mathrm{H} \alpha$ flux, the coronal X-ray flux, and the accretion-line flux, suggesting an origin distinct from the accretion process. While assignment of these variations to 
short-lived hot spots on the stellar surface has been offered by many (Huélamo et al. 2008; Mekkaden 1998; Batalha et al. 2002), other authors (Rucinski et al. 2008; Siwak et al. 2011) have conjectured that changing optical periods may be associated with Keplerian rotation of "blobs" of large magnetically controlled structures in the circumstellar disk. Our observations appear to eliminate photometric variability directly associated with the accretion process; we cannot discriminate among other possibilities.

2. The $\mathrm{H} \alpha$ profile appears to be intrinsically symmetric and the short- and long-wavelength sides of the emission are correlated. We suggest this broad line and $\mathrm{H} \beta$ as well arise in the turbulent post-shock cooling region, and not, as frequently assumed from the funneled accretion flow from the circumstellar disk to the star as a "ballistic infall signature." The $\mathrm{H} \beta$ line appears to be a superior diagnostic of dynamics because it is less optically thick than $\mathrm{H} \alpha$. Both of these profiles are substantially modified by a wind originating from the star that increases in optical depth over several days. These changes may be driven by the X-ray accretion or the rotation of the star presenting different aspects of an asymmetric outflow over the polar regions. Modeling of the optical line profiles must recognize the complex origin of the emissions and modifications by the stellar outflows. The presence of a turbulent cooling region offers a straightforward explanation of the broad permitted emission lines including those in the UV and far-UV regions (Herczeg et al. 2002; Dupree et al. 2005).

3. The temporal variation of the He I $5876 \AA$ line profile in addition to its correspondence in width with other species suggests that the post-shock cooling region also produces the broad component of the profile.

4. This study clearly reveals the sequence of the accretion process in various energy bands and spectral lines. Enhanced accretion, inferred from the $\mathrm{X}$-ray accretion-line fluxes, is followed by inflow signatures in $\mathrm{H} \alpha, \mathrm{H} \beta$, and an increased flux in the broad wing of the He I ( $5876 \AA$ ) line arising in the turbulent post-shock cooling region. Subsequently the photospheric veiling increases, and the response of the stellar corona $2.5 \mathrm{hr}$ later, follows the increase in the photospheric veiling.

5. Spectroscopy gives direct evidence for the influence of the accretion process in heating the corona and provides observational confirmation of simulations of coronal heating and wave-driven winds (Cranmer 2008, 2009).

We are grateful to Jonathan Irwin for assistance with the WASP data. The WASP consortium comprises the University of Cambridge, Keele University, University of Leicester, The Open University, The Queens University Belfast, St. Andrews University, and the Isaac Newton Group. Funding for WASP comes from the consortium universities and from the Science and Technology Facilities Council of the UK. We appreciate the efforts of Luca DiFabrizio who reduced the TNG/SARG spectrum. This paper includes data gathered with the $6.5 \mathrm{~m}$ Magellan Telescopes located at Las Campanas Observatory, Chile. Also, based on observations obtained at the Gemini Observatory, which is operated by the Association of Universities for Research in Astronomy, Inc., under a cooperative agreement with the NSF on behalf of the Gemini partnership: the National Science Foundation (United States), the Science and Technology Facilities Council (United Kingdom), the National Research Council (Canada), CONICYT (Chile), the Australian

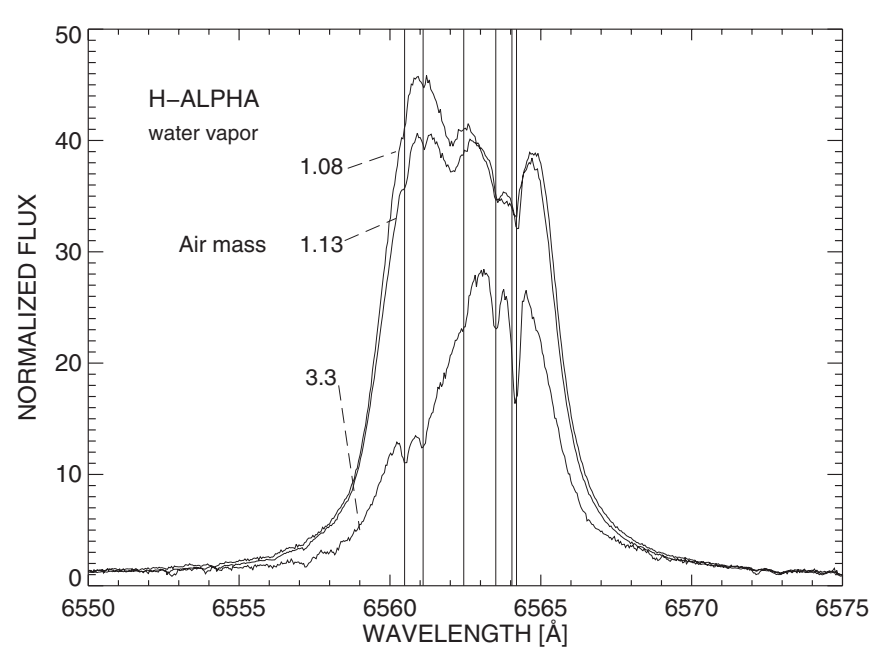

Figure 22. Three profiles of $\mathrm{H} \alpha$ to illustrate the presence of water vapor in the profile. Spectra were taken at three different air masses and are aligned on the rest wavelengths of the water vapor as determined in the HITRAN database (Rothman et al. 2009).

Research Council (Australia), Ministério da Ciência, Tecnologia e Inovação (Brazil), and Ministerio de Ciencia, Tecnología e Innovación Productiva (Argentina).

Facilities: ASAS (U. Warsaw), CXO (HETG), Gemini: South (PHOENIX), Keck:II (NIRSPEC), Magellan:Clay (MIKE), Radcliffe (Cassegrain spectrograph), ATT (echelle), LNA:1.6m(coudé), TNG (SARG), VBT (echelle), SuperWASP

\section{APPENDIX}

\section{EFFECT OF WATER VAPOR}

Water vapor absorption in Earth's atmosphere occurs at wavelengths overlapping the $\mathrm{H} \alpha$ profile, but only in severe instances does it affect the total line flux. Examples of absorption in the emission profile are shown in the accompanying figure. Wavelengths are taken from the HITRAN database (Rothman et al. 2009). The strongest line in this region estimated from the R. Kurucz synthesis of the solar spectrum occurs at $6564.195 \AA$. It is clear that the peak in the $\mathrm{H} \alpha$ profile at the longest wavelengths can be affected by water vapor absorption, although the lines are narrow. The three profiles in Figure 22 show that the wind absorption is dominant in shaping the overall profile. The spectrum at the highest air mass of 3.3 was taken under adverse conditions during the monsoon season in India.

\section{REFERENCES}

Alencar, S. H. P., \& Batalha, C. 2002, ApJ, 571, 378

Ardila, D. R., Basri, G., Walter, F. M., Valenti, J. A., \& Johns-Krull, C. M. 2002, ApJ, 567, 1013

Batalha, C., Batalha, N. M., Alencar, S. H. P., Lopes, D. F., \& Duarte, E. S. 2002, ApJ, 580, 343

Bernstein, R., Shectman, S. A., Gunnels, S. M., Mochnacki, S., \& Athey, A. E. 2003, Proc. SPIE, 4841, 1694

Bertout, C. 1989, ARA\&A, 27, 351

Brickhouse, N. S., Cranmer, S. R., Dupree, A. K., Luna, G. J. M., \& Wolk, S. 2010, ApJ, 710, 1835

Butters, O. W., West, R. G., Anderson, D. R., et al. 2010, A\&A, 520, L10

Costa, V. M., Lago, M. T. V. T., Norci, L., \& Meurs, E. J. A. 2000, A\&A, 354, 621

Cranmer, S. R. 2008, ApJ, 689, 316

Cranmer, S. R. 2009, ApJ, 706, 824

Curran, R. L., Argiroffi, C., Sacco, G. G., et al. 2011, A\&A, 526, A104 
Donati, J.-F., Gregory, S. G., Alencar, S. H. P., et al. 2011, MNRAS, 417, 472

Dupree, A. K., Brickhouse, N. S., Smith, G. H., \& Strader, J. 2005, ApJ, 625, L131

Edwards, S., Fischer, W., Kwan, J., Hillenbrand, L., \& Dupree, A. K. 2003, ApJ, 599, L41

Günther, H. M., \& Schmitt, J. H. M. M. 2008, A\&A, 481, 735

Hartigan, P., Hartmann, L., Kenyon, S., Hewett, R., \& Stauffer, J. 1989, ApJS, 70,899

Hartmann, L., Hewett, R., \& Calvet, N. 1994, ApJ, 426, 669

Herczeg, G. J., Linsky, J. L., Valenti, J. A., Johns-Krull, C. M., \& Wood, B. E. 2002, ApJ, 572, 310

Horne, J. H., \& Baliunas, S. L. 1986, ApJ, 302, 757

Huélamo, N., Figueira, P., Bonfils, X., et al. 2008, A\&A, 489, L9

Hummer, D. G., \& Rybicki, G. B. 1968, ApJ, 153, L107

Kastner, J. H., Huenemoerder, D. P., Schulz, N. S., Canizares, C. R., \& Weintraub, D. A. 2002, ApJ, 567, 434

Kastner, J. H., Huenemoerder, D. P., Schulz, N. S., \& Weintraub, D. A. 1999, АpJ, 525, 837

Koen, C., \& Eyer, L. 2002, MNRAS, 331, 45

Krist, J. E., Stapelfeldt, K. R., Ménard, F., Padgett, D. L., \& Burrows, C. J. 2000, ApJ, 538, 793

Kurosawa, R., Romanova, M. M., \& Harries, T. J. 2011, MNRAS, 416, 2623

Lamzin, S. A., Romanova, M. M., \& Kravtsova, A. S. 2007, in Proc. IAU 3, Symp. 243, Star-Disk Interaction in Young Stars, ed. J. Bouvier \& I. Appenzeller (Cambridge: Cambridge Univ. Press), 115

Lawson, W. A., \& Crause, L. A. 2005, MNRAS, 357, 1399

Lawson, W. A., Crause, L. A., Mamajek, E. E., \& Feigelson, E. D. 2001, MNRAS, 321, 57
Mallik, S. V., Brickhouse, N. S., \& Dupree, A. K. 2010, in Recent Advances in Spectroscopy Theoretical, Astrophysical, and Experimental Perspectives, ed. R. K. Chaudhuri, M. V. Mekkaden, A. V. Raveendran, \& A. S. Narayanan (Berlin: Springer), 193

Mekkaden, M. V. 1998, A\&A, 340, 135

Muzerolle, J., Calvet, N., Briceño, C., Hartmann, L., \& Hillenbrand, L. 2000, ApJ, 535, L47

Muzerolle, J., Calvet, N., \& Hartmann, L. 1998a, ApJ, 492, 743

Muzerolle, J., Calvet, N., \& Hartmann, L. 2001, ApJ, 550, 944

Muzerolle, J., Hartmann, L., \& Calvet, N. 1998b, AJ, 116, 455

Natta, A., Testi, L., Muzerolle, J., et al. 2004, A\&A, 424, 603

Pojmanski, G. 2002, Acta Astron., 52, 397

Qi, C., Ho, P. T. P., Wilner, D. J., et al. 2004, ApJ, 616, L11

Romanova, M. M., Ustyugova, G. V., Koldoba, A. V., \& Lovelace, R. V. E. 2004, ApJ, 610, 920

Rothman, L. S., Gordon, I. E., Barbe, A., et al. 2009, J. Quant. Spectrosc. Radiat. Transfer, 110, 533

Rucinski, S. M., \& Krautter, J. 1983, A\&A, 121, 217

Rucinski, S. M., Matthews, J. M., Kuschnig, R., et al. 2008, MNRAS, 391, 1913

Sacco, G. G., Orlando, S., Argiroffi, C., et al. 2010, A\&A, 522, A55

Scargle, J. D. 1982, ApJ, 263, 835

Siwak, M., Rucinski, S. M., Matthews, J. M., et al. 2011, MNRAS, 410, 2725

Stassun, K. G., van den Berg, M., \& Feigelson, E. 2007, ApJ, 660, 704

Stassun, K. G., van den Berg, M., Feigelson, E., \& Flaccomio, E. 2006, ApJ, 649,914

Vacca, W. D., \& Sandell, G. 2011, ApJ, 732, 8

White, R. J., \& Basri, G. 2003, ApJ, 582, 1109

Wichmann, R., Bastian, U., Krautter, J., Jankovics, I., \& Rucinski, S. M. 1998, MNRAS, 301, 39L

Yang, H., Johns-Krull, C. M., \& Valenti, J. A. 2007, AJ, 133, 73 